\title{
Fluid-particle dynamics in canalithiasis
}

\author{
Dominik Obrist $^{1, *}$ and Stefan Hegemann ${ }^{2}$ \\ ${ }^{1}$ Institute of Fluid Dynamics, ETH Zurich, 8092 Zurich, Switzerland \\ ${ }^{2}$ Department of Otorhinolaryngology, University Hospital Zurich, 8091 Zurich, Switzerland
}

\begin{abstract}
The semicircular canals (SCCs; located in the inner ear) are the primary sensors for angular motion. Angular head movements induce a fluid flow in the SCCs. This flow is detected by afferent hair cells inside the SCCs. Canalithiasis is a condition where small particles disturb this flow, which leads to benign paroxysmal positional vertigo (top-shelf vertigo).

The present work investigates the interaction between the fluid flow and the particles on the basis of an idealized analytical model. Numerical solutions of the full model and a thorough analytical study of the linearized model reveal the principal mechanisms of canalithiasis. We propose a set of dimensionless numbers to characterize canalithiasis and derive explicit expressions connecting these dimensionless numbers directly to the typical clinical symptoms.
\end{abstract}

Keywords: semicircular canal; canalithiasis; benign paroxysmal positional vertigo

\section{INTRODUCTION}

Benign paroxysmal positional vertigo (also known as $B P P V$ or top-shelf vertigo) accounts for $20-30 \%$ of all vertigo syndromes in humans (Baloh et al. 1989). BPPV is suspected to be caused by small calcite particles floating freely in the semicircular canals (SCCs) of the vestibular system located in the inner ear. This condition, known as canalithiasis, is the main subject of the present investigation.

The SCCs are the primary sensors for angular motion. They form a system of slender ducts oriented in mutually orthogonal planes that are filled with endolymph (a fluid with properties similar to water). At one end, the SCCs are open and connected with the utricle. At the other end, a short section of the duct is widened (ampulla). This section contains the sensory hair cell receptors that are embedded in a gelatinous structure called the cupula. The cupula spans the complete lumen of the SCC. Thus, it functionally plugs the canal. Axial flow of the endolymph deflects the cupula like the wind bulges a sail. When the cupula is deflected, the sensory hair cells increase or decrease their firing rate depending on the orientation of the deflection. The brain determines the direction of the head rotation by vectorial summation of the inputs of all six SCCs (from the left and right ear), which leads to the perception of angular motion. The perceived velocity signal leads to an eye movement that compensates for the head movement such that we are able to maintain our focus on an object while moving. During continuous motion in the same direction, the eyes have to be reset at some point by quick eye movements. The repetitive pattern of slow compensatory movements (slow phases) and quick resetting

*Author for correspondence (obrist@ifd.mavt.ethz.ch). movements (quick phases) is called nystagmus. We call the velocity of the compensatory eye movements (slow phase) the nystagmus velocity $N$. It is known that the nerve signal from the cupula is controlled and altered by several intricate mechanisms of the central nerve system before it is translated to the ocular muscles (Cohen et al. 1977; Robinson 1977; Raphan et al. 1979). Squires et al. (2004), for example, included velocity storage in their model for top-shelf vertigo. Owing to the velocity storage mechanism, the time constant for the decay of the nystagmus velocity is considerably higher than that for the SCC itself (the time constant of the SCC will be discussed in some more detail in $\$ 2$ ). Squires et al. (2004) stated, however, that their fundamental analysis of the mechanical processes in the SCC is not affected by the velocity storage. Therefore, we assume for the scope of this work that $N$ is proportional to $V$, where $V$ is the volumetric displacement of the cupula

$$
V(t)=\int^{t} \iint_{A_{\mathrm{c}}} u \mathrm{~d} A \mathrm{~d} \tau,
$$

with $u$ and $A_{\mathrm{c}}$ denoting the axial velocity component of the endolymph flow and the cross-sectional area of the canal, respectively.

Figure 1 shows the nystagmus velocity (vertical component) of a patient with BPPV with canalithiasis during and after a head manoeuvre. In this clinical experiment, performed by the second author, the eye movements were recorded in a magnetic frame (Remmel-type system, modified by Lasker, Baltimore) using dual scleral search coils (Skalar Instruments, Delft). A search coil was placed on the right eye around the cornea (after anaesthetizing the conjunctiva with oxybuprocaine $0.4 \%$ ). The angular velocity was computed according to Hepp (1990) and Tweed et al. (1990). During the movement of the head 


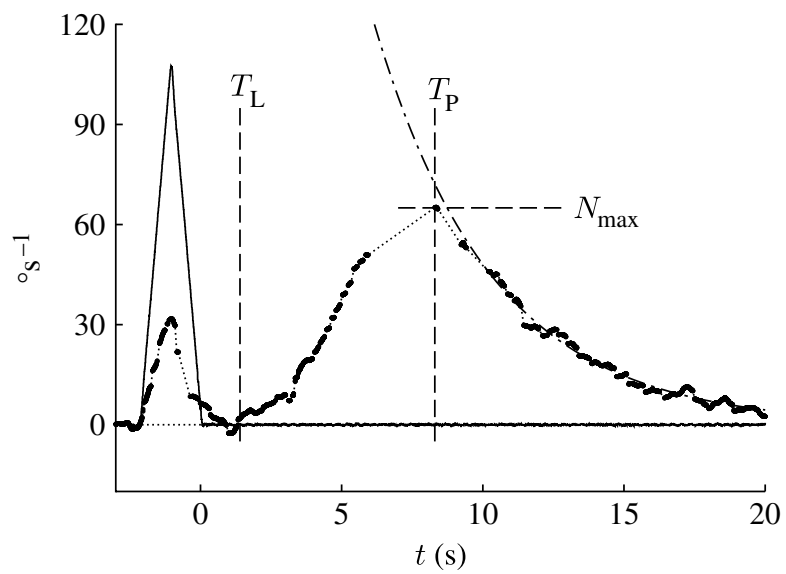

Figure 1. Nystagmus velocity $N$ (dots) and head velocity (solid line) of a canalithiasis patient during and after a head manoeuvre. The time $T_{\mathrm{P}}$ and the magnitude $N_{\max }$ of the maximum nystagmus velocity are only estimates due to incomplete data between $t=6$ and $10 \mathrm{~s}$. After its peak $(t>10 \mathrm{~s})$, the positional nystagmus decays approximately like $\exp (-t / 4.2 \mathrm{~s})$ (dot-dashed line).

$(-3 \mathrm{~s}<t<0 \mathrm{~s})$, the nystagmus velocity $N$ is approximately proportional to the head velocity. This is called the per-rotatory nystagmus, which is the normal reaction to an angular head movement. It is symptomatic for canalithiasis patients that there is a second nystagmus after a certain onset latency $T_{\mathrm{L}}$. We call this the positional nystagmus. It is pathological and causes vertigo. The onset latency $T_{\mathrm{L}}$, the time to peak $T_{\mathrm{P}}$ and the maximum strength of the nystagmus $N_{\max }$ are the characteristic values of a positional nystagmus. In clinical testing, it is found that these values may differ substantially between different patients, different head manoeuvres or even different instances of the same experiment. The onset latency, for instance, typically lasts for a few seconds, but there are also cases where the positional nystagmus starts immediately after the head manoeuvre. The total duration of the positional nystagmus ranges typically from 10 to $60 \mathrm{~s}$ (Aw et al. 2005). A priori, it is not clear whether these different characteristic values are related to different sizes or numbers of particles in the SCC. However, we will present results that show that different particle sizes and numbers have a direct effect on the duration and intensity of the positional nystagmus, and we will show that certain particle sizes and numbers may lead to a vanishing onset latency.

Theoretical work on the fluid mechanics of SCC dates back to Breuer (1874), Crum Brown (1874) and Mach (1875), who were the first authors to connect the SCC to fluid mechanics. More recently, Van Buskirk \& Grant (1973) and later Van Buskirk et al. (1976) proposed a model for the flow of the endolymph in the SCC based on the Navier-Stokes equations. They derived a partial integro-differential equation that describes the axial velocity profile. The restoring force of the deflected cupula enters this equation in the form of a double integral similar to (1.1). We will return to their results in §2. Rabbitt \& Damiano (1992) and Damiano \& Rabbitt (1996) worked with intricate methods of matched asymptotics to describe the fluid dynamics of SCC in great detail. Their results confirmed some of the findings of Van Buskirk et al. and other authors. They provided a detailed understanding of the flow not only in the SCC but also in the ampulla. Ifediba et al. (2007) used these results to simulate and study the biomechanics of the complete three-canal system. Obrist (2008) based his study on the equation for a single SCC proposed by Van Buskirk et al. Whereas the latter used asymptotics to solve the equation, Obrist found an exact solution to the problem. He analysed the eigenvalue spectrum of the model and used a modal expansion to give an explicit expression for the cupula displacement as a function of the head manoeuvre. The present work builds on these results.

Theoretical work on BPPV is still relatively sparse. Only recently papers by House \& Honrubia (2003), Rajguru et al. (2004, 2005) and Squires et al. (2004) brought forward mathematical models for BPPV. The aim of the present work is to reveal the principal mechanisms leading to the positional nystagmus. We will identify the important parameters and their relationship with characteristic features of canalithiasis (e.g. onset latency, time to peak and peak nystagmus velocity).

In contrast to earlier investigations, we deliberately choose an idealized geometry limited to a single SCC with constant circular cross section in the slender part. This is in strong contrast to the work of Squires et al. (2004), which heavily relies on the varying cross section of the SCC. It is also different from Rajguru et al. (2004, 2005), who used a detailed three-dimensional model of all three SCCs that allowed them to study complex head manoeuvres. Here, we aim to idealize the problem as much as possible and to retain as little as necessary, which will allow us to study the principal mechanisms in great detail. Our idealized model is able to offer the following: clear explanations for certain phenomena; explicit expressions for the characteristic features of the positional nystagmus; and the dimensionless numbers that are most relevant to canalithiasis.

Established results on the fluid dynamics of SCC (without particles) are summarized in $§ 2$. We will derive a fluid-particle model with two-way coupling in $\S 3$. The governing equations feature several dimensionless coefficients that indicate the relevance of the different physical phenomena. Numerical solutions of the governing equations are studied in $\S 4$, which will explain the central mechanisms of canalithiasis. We will describe a mechanism for the onset latency. In contrast to the explanation offered by Squires et al. (2004), this mechanism does not require the particles to be located within the ampulla at the beginning of the manoeuvre. Also, it will be shown that the onset latency may vanish under certain conditions. In addition, §4 includes a parameter study to illustrate the influence of size and number of particles on the positional nystagmus. To justify our one-dimensional particle model, we compare our numerical results with those obtained from a more sophisticated particle model and show that the fundamental features of canalithiasis are sufficiently well represented by our particle model. In $\$ 5$, a linearized version of the governing equations is analysed in detail. We will find analytical approximations for the temporal 


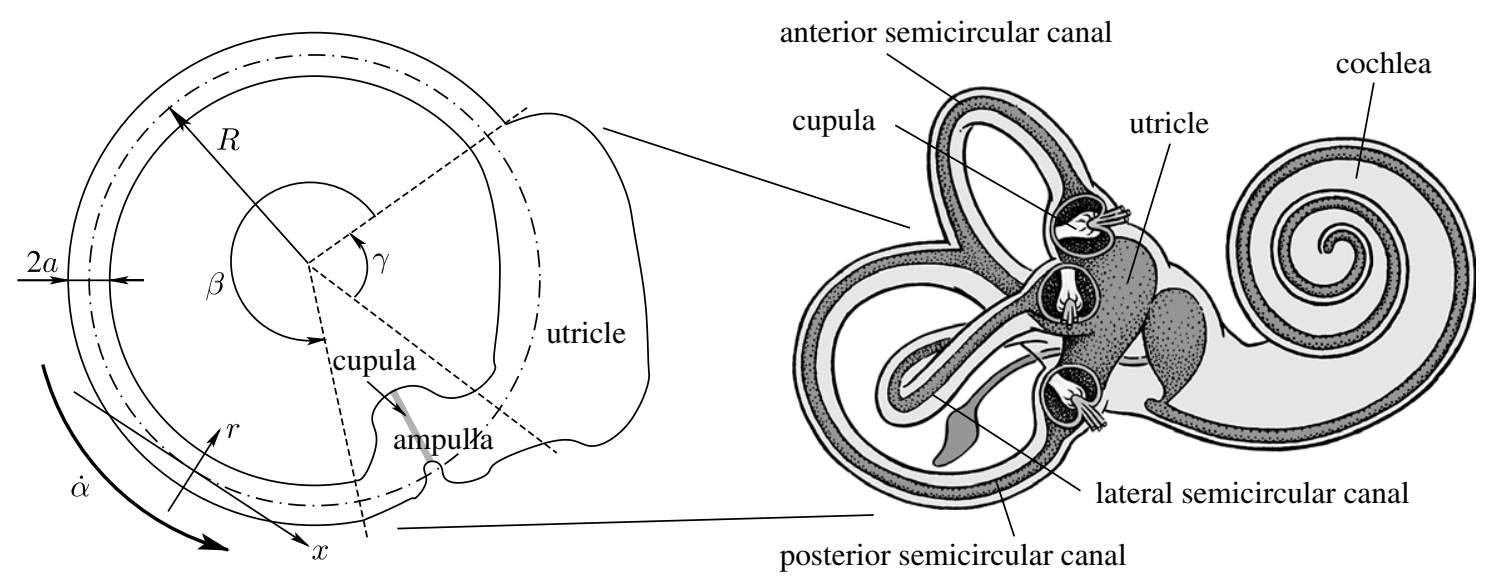

Figure 2. The human vestibular system with schematic of a single SCC. (Reproduced from an original illustration by M. Pinker.)

eigenvalues. From this we will define a Stokes number that relates the particle size directly to the positional nystagmus. Later in this section, explicit relationships between the cupula displacement and the particle properties are derived. These relationships show that the Stokes number is one of the most relevant parameters for canalithiasis. We will demonstrate how these relationships might be used to diagnose the particle size from clinical data. Section 6 concludes this paper.

\section{FLUID DYNAMICS OF SEMICIRCULAR CANALS}

This section gives a brief summary of the established results on the fluid dynamics of healthy SCCs without any particles. Most of the results in this section were derived by Van Buskirk et al. (1976) and Obrist (2008).

The model of Van Buskirk et al. considers a single idealized SCC (figure 2) under angular head movements $\alpha(t)$. This model describes only the flow in the slender part of the SCC that spans an angle $\beta$ and has a constant circular cross section $A_{\mathrm{c}}=\pi a^{2}$. The radius of the cross section $a$ is much smaller than the major radius $R$ of the torus. The endolymph in the utricle (which spans the angle $\gamma$ ) enters the equation only as an inertial force (Van Buskirk 1977). The cupula is modelled by a restoring force that is proportional to the volume displacement $V(t)$ of the cupula. More complex nonlinear models for the cupula (e.g. Rabbitt \& Damiano 1992; Yamauchi et al. 2001) will not be considered here.

Under these assumptions, the axial component of the Navier-Stokes equations for the flow in the SCC becomes (Van Buskirk et al. 1976)

$$
\begin{aligned}
\dot{u} & -\frac{\nu}{r} \frac{\partial}{\partial r}\left[r \frac{\partial}{\partial r} u\right]+\frac{2 \pi K}{\rho \beta R} \int_{0}^{t} \int_{0}^{a} u\left(r^{\prime}, \tau\right) r^{\prime} \mathrm{d} r^{\prime} \mathrm{d} \tau \\
& =-(1+\gamma / \beta) R \ddot{\alpha}(t),
\end{aligned}
$$

with boundary conditions

$$
u(r=a)=\frac{\partial}{\partial r} u(r=0)=0,
$$

where the axial velocity $u(r, t)$ depends on the time $t$ and the radial variable $r$, which goes from 0 (centreline)
Table 1. Typical physical and geometrical parameters (Van Buskirk et al. 1976; Squires et al. 2004).

\begin{tabular}{ll}
\hline description & value \\
\hline major canal radius, $R$ & $3.2 \times 10^{-3} \mathrm{~m}$ \\
duct radius, $a$ & $1.6 \times 10^{-4} \mathrm{~m}$ \\
angle spanned by the duct, $\beta$ & $1.4 \pi$ \\
angle spanned by the utricle, $\gamma$ & $0.42 \pi$ \\
cupular stiffness, $K$ & $13 \mathrm{GPa} \mathrm{m}^{-3}$ \\
endolymph density, $\rho$ & $10^{3} \mathrm{~kg} \mathrm{~m}^{-3}$ \\
endolymph viscosity, $\nu$ & $10^{-6} \mathrm{~m}^{2} \mathrm{~s}^{-1}$ \\
particle density, $\rho_{\mathrm{p}}$ & $2.7 \times 10^{3} \mathrm{~kg} \mathrm{~m}^{-3}$ \\
particle radius, $a_{\mathrm{p}}$ & $0.5-15 \mu \mathrm{m}$ \\
\hline
\end{tabular}

to $a$ (at the wall). Throughout this paper, we use as a shorthand notation for the derivative with respect to the dimensional time $t$. See table 1 for a list of physical and geometrical parameters used in this work. (A detailed derivation of Van Buskirk's equation can be found in appendix A.)

We arrive at the dimensionless form of $(2.1 a)$ and (2.1b) by defining

$$
\tilde{r}=\frac{r}{a}, \quad \tilde{t}=\frac{t}{T_{\text {ref }}}, \quad \tilde{u}(\tilde{r}, \tilde{t})=\frac{T_{\text {ref }}}{R} u(r, t),
$$

where $T_{\text {ref }}=a^{2} / \nu$ is the viscous time scale. The viscous time scale $\left(T_{\text {ref }} \approx 0.026 \mathrm{~s}\right.$ ) determines how fast viscous phenomena attain their steady state. Therefore, all dynamical processes that are much slower than $T_{\text {ref }}$ can be considered quasi-steady with respect to viscous effects. With (2.2), we obtain

$$
\begin{aligned}
\frac{\partial}{\partial \tilde{t}} \tilde{u}-\frac{1}{\tilde{r}} \frac{\partial}{\partial \tilde{r}} & \left(\tilde{r} \frac{\partial}{\partial \tilde{r}} \tilde{u}\right)+\epsilon \int_{0}^{\tilde{t}} \int_{0}^{1} \tilde{u}\left(r^{\prime}, t^{\prime}\right) r^{\prime} \mathrm{d} r^{\prime} \mathrm{d} t^{\prime} \\
& =-(1+\gamma / \beta) \frac{\partial^{2}}{\partial \tilde{t}^{2}} \alpha,
\end{aligned}
$$

with the dimensionless cupula stiffness $\epsilon$,

$$
\epsilon=\frac{2 A_{\mathrm{c}} K T_{\mathrm{ref}}^{2}}{\rho \beta R} .
$$

The coefficient $\epsilon$ is proportional to the mechanical stiffness $K$ of the cupula. A stiffer cupula, i.e. a higher $\epsilon$, 


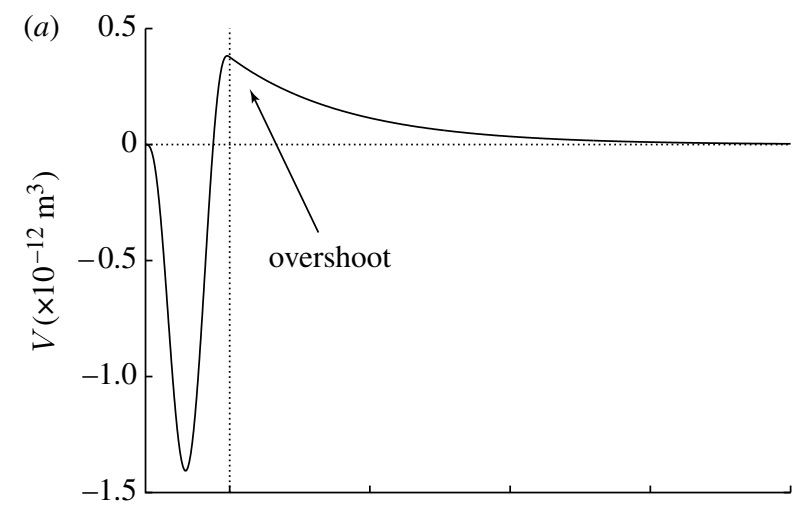

(b)

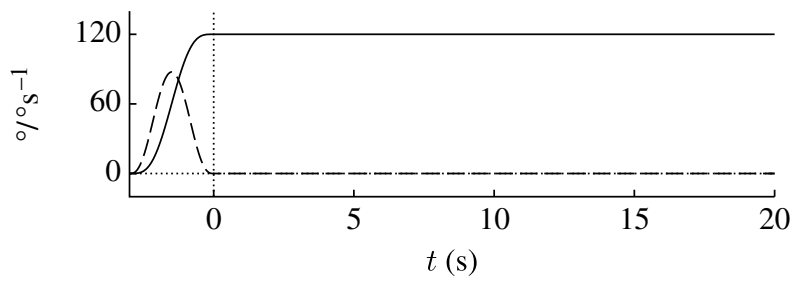

Figure 3. (a) Cupular displacement $V(t)$ in an SCC without particles during and after $(b)$ a head manoeuvre from $\alpha=0^{\circ}$ to $120^{\circ}\left(\epsilon=0.09752, \sigma_{\mathrm{c}} \approx 0.00610\right)$. Solid line, $\alpha(t)$; dashed line, $\dot{\alpha}(t)$.

leads to a faster relaxation of the deflected cupula. With the parameters from table 1 , we get $\epsilon=0.09752$ (this value will be used hereafter unless noted otherwise).

The ansatz $\tilde{u}(\tilde{r}, \tilde{t})=\hat{u}(\tilde{r}) \mathrm{e}^{-\sigma \tilde{t}}$ yields the eigenmodes of (2.3). The associated eigenvalues $\sigma$ are real and positive, i.e. all modes decay in time and do not oscillate. The least stable mode is directly related to the mechanical properties of the cupula and is named cupula mode. Its eigenvalue is approximately

$$
\sigma_{\mathrm{c}} \approx \frac{\epsilon}{16}
$$

and its eigenfunction is close to the parabola $1-r^{2}$. The cupula relaxes after a head manoeuvre (cf. figure 3) according to this eigenfunction like $\exp (-\epsilon \tilde{t} / 16)$. This corresponds approximately to $\exp (-t / 4.2 \mathrm{~s})$ where $4.2 \mathrm{~s}$ is a typical time constant for the lateral SCC (Dai et al. 1999). The other modes depend only weakly on $\epsilon$. They are called duct modes. Their eigenvalues are approximately given by $\sigma \approx \lambda_{k}^{2}$ where $\lambda_{k}$ is the $k$ th root of the Bessel function $J_{0}$. All duct modes decay very rapidly with a time constant much smaller than $T_{\text {ref }}$.

Obrist (2008) showed that the cupula displacement is given by

$$
\begin{aligned}
V(t) \approx & -A_{\mathrm{c}} R T_{\mathrm{ref}} \frac{1+\gamma / \beta}{8} \\
& \times\left[\dot{\alpha}(t)-\sigma_{\mathrm{c}} \int_{0}^{\infty} \dot{\alpha}\left(t-\tau T_{\text {ref }}\right) \mathrm{e}^{-\sigma_{\mathrm{c}} \tau} \mathrm{d} \tau\right],
\end{aligned}
$$

where the factor before square brackets amounts to approximately $1.07 \times 10^{-12} \mathrm{~m}^{3} \mathrm{~s}$ (using the values from table 1). This equation is approximate in the sense that it yields accurate results for the head manoeuvres with angular velocities that change slowly with respect to the viscous time scale $T_{\text {ref. }}$ For most natural head manoeuvres, (2.6) is sufficiently accurate.
Equation (2.6) is analytical evidence that SCCs are indeed good sensors for angular motion, since it shows that $V \propto \dot{\alpha}$ apart from the velocity error

$$
\dot{\alpha}_{\mathrm{e}}=-\sigma_{\mathrm{c}} \int_{0}^{\infty} \dot{\alpha}\left(t-\tau T_{\mathrm{ref}}\right) \mathrm{e}^{-\sigma_{\mathrm{c}} \tau} \mathrm{d} \tau .
$$

If the velocity error is strong enough, it may lead to a sensation of reverse angular motion after the actual angular motion has ended. In any case, the velocity error leads to an overshoot in the cupula displacement at the end of a head manoeuvre. Figure 3 demonstrates this effect by numerical integration of (2.3) for a smooth head rotation from an upright $\left(0^{\circ}\right)$ to a supine position $\left(120^{\circ}\right)$ in $3 \mathrm{~s}$. Such a head manoeuvre is part of a diagnostic sequence known as the Dix-Hallpike manoeuvre (Dix \& Hallpike 1952). It is often used in clinical experiments (figure 1) and we will use it throughout this work.

Figure 3 shows that the cupula displacement $V(t)$ is nearly proportional to the angular head velocity $\dot{\alpha}(t)$ during the manoeuvre. At $t=0 \mathrm{~s}$ when the manoeuvre has finished, the cupula has overshot its relaxed position and returns only slowly (with time constant $T_{\text {ref }} / \sigma_{\mathrm{c}} \approx 4.2 \mathrm{~s}$ ) to its relaxed position.

\section{GOVERNING EQUATIONS FOR THE SEMICIRCULAR CANAL WITH PARTICLES}

The governing equations for the particulate flow in the SCC are based on Van Buskirk's equation (2.1a) and $(2.1 b)$, to which we add an equation for the particle motion. In addition, we introduce coupling terms to model the fluid-particle interaction.

The modelling of the particle dynamics is a critical issue. The particle velocities and trajectories have a direct impact on the endolymph flow and on the positional nystagmus. The particles are calcite particles (otoconia) presumably broken off the macula of the utricle. Campos (1990) reported radii ranging from 0.5 to $15 \mu \mathrm{m}$. However, the actual number, size and shape of particles in the SCC of canalithiasis patients is not known, and it is probable that the particles are nonspherical and that collisions between particles will occur. It is also clear that the particles will directly interact with the wall at some point. Whether they are likely to stick to the wall or whether they will roll or slide along the wall is not known. An accurate prediction of the particle trajectories is obviously very difficult. Nevertheless, it is possible to make some reasonable idealizations that allow us to understand the principal mechanisms of canalithiasis.

Squires et al. (2004) used a two-dimensional particle model that keeps track of the radial and axial positions of a particle. Their model is based on asymptotic results for small spheres sedimenting in circular pipes under Stokes flow conditions (Bungay \& Brenner 1973; Happel \& Brenner 1973). In this model, the pressure drop due to a sedimenting particle is the highest if the particle falls along the centreline of the pipe. As the particle approaches the wall, the pressure decreases. Furthermore, Squires et al. introduced a lubrication gap between the particle and the wall 
$(1 \mu \mathrm{m})$ to prevent the particles from sticking to the wall. This lubrication gap allows the particles to slide along the wall with 30 per cent or more of their freefall velocity. At the same time, Squires et al. assumed that the pressure drop due to particles is negligibly small if they slide along the wall.

Rajguru \& Rabbitt (2007) showed recently in an experimental study that the hair cells in the cupula are also stimulated by particles that are sliding along the wall. They explain this apparent contradiction between their experiment and the assumptions of Squires et al. with the size of the particles: whereas the model of Squires et al. is derived for small particles, Rajguru \& Rabbitt used relatively large particles (more than $10 \%$ of the canal diameter). This suggests that the pressure drop due to (larger) particles sliding along the wall is not negligible.

A more pragmatic approach to particle modelling was taken by House \& Honrubia (2003) and Rajguru et al. (2004). They formulated a one-dimensional particle model based on Stokes' formula for the drag of a sphere in an unbounded fluid. This model tracks only the axial position of the particles in the SCC. The most striking difference between the two approaches lies in the pressure drop. Whereas the pressure drop of Squires et al. varied strongly with respect to the radial position, the pressure drop of House \& Honrubia is independent of the radial position. If the particle is in the centre of the canal, the model of House \& Honrubia underpredicts the pressure drop by a factor 2 , and it overpredicts the pressure drop if the particle is close to the wall. Averaged over the circular cross section, however, the pressure drop is equal for both models. In this light, we can expect that (on average) the two models should give similar predictions for the cupula displacement if we assume that a particle passes through different radial positions during the course of a positional nystagmus. In the present work, we assume (for now) that the one-dimensional particle model is sufficiently accurate for a study of the principal mechanisms of canalithiasis. We will justify this assumption in $\$ 4.1$ by comparing numerical results obtained with both particle models.

For the formulation of our one-dimensional particle model, we consider $n_{\mathrm{p}}$ particles of radius $a_{\mathrm{p}}$ and mass $m_{\mathrm{p}}$ which move freely in an SCC. Their motion is governed by the particle inertia, the Stokes drag of the particle, the particle gravity and an external forcing (angular motion of the head). Their axial position is denoted by $x_{\mathrm{p}}(t)$ (measured relative to the SCC). The origin $x_{\mathrm{p}}=0$ is set to the (initially) lowest point in the canal

$$
x_{\mathrm{p}}(0)=\dot{x}_{\mathrm{p}}(0)=0 \text {. }
$$

The particle equation of motion,

$$
m_{\mathrm{p}}\left(\ddot{x}_{\mathrm{p}}+R \ddot{\alpha}\right)=F_{\mathrm{s}}+F_{\mathrm{g}},
$$

includes a term for the particle inertia $m_{\mathrm{p}}\left(\ddot{x}_{\mathrm{p}}+R \ddot{\alpha}\right)$ and two terms for external forces, the gravitational force

$$
F_{\mathrm{g}}=-m_{\mathrm{p}}\left(1-\rho / \rho_{\mathrm{p}}\right) g \sin \left(x_{\mathrm{p}} / R+\alpha\right),
$$

and a Stokes drag force for a sphere of radius $a_{\mathrm{p}}$

$$
F_{\mathrm{s}}=-6 \pi \nu \rho a_{\mathrm{p}}\left(\dot{x}_{\mathrm{p}}-u_{p}\right) .
$$

The velocity $u_{\mathrm{p}}$ is the local fluid velocity at the particle location. Since we do not keep track of the radial position, we approximate the $u_{\mathrm{p}}$ by the bulk velocity of the fluid

$$
u_{\mathrm{p}}=\frac{2 \pi}{A_{\mathrm{c}}} \int_{0}^{a} u\left(r^{\prime}, t\right) r^{\prime} \mathrm{d} r^{\prime} .
$$

At the same time, we introduce the Stokes drag (3.4) to the fluid equation $(2.1 a)$ and $(2.1 b)$. We assume that the Stokes drag $F_{\mathrm{s}}$ of $n_{\mathrm{p}}$ particles induces an axial pressure gradient $-n_{\mathrm{p}} F_{\mathrm{s}} /\left(A_{\mathrm{c}} \beta R\right)$ along the slender duct.

Again, we use the dimensionless variables (2.2) and $\tilde{x}_{\mathrm{p}}(\tilde{t})=x_{\mathrm{p}}(t) / R$. Furthermore, we define the Froude number $F r$ as

$$
F r^{2}=\frac{R}{g \mathrm{~T}_{\mathrm{ref}}^{2}\left(1-\rho / \rho_{\mathrm{p}}\right)} .
$$

The Froude number is a dimensionless coefficient occurring frequently in problems that involve fluid dynamics and gravity. It relates inertial forces $\left(\sim a_{\mathrm{p}}^{3} \rho_{\mathrm{p}} R / T_{\text {ref }}^{2}\right)$ to gravity forces $\left(\sim a_{\mathrm{p}}^{3}\left(\rho_{\mathrm{p}}-\rho\right) g\right)$. For the parameters from table 1 , we get $F^{2}=0.79052$. Since $\mathrm{Fr}^{2}=O(1)$ the inertial and gravity forces play an equally important role in canalithiasis. With these definitions, the governing equations for the particulate flow in an SCC are

$$
\begin{aligned}
& \frac{\partial}{\partial \tilde{t}} \tilde{u}-\frac{1}{\tilde{r}} \frac{\partial}{\partial \tilde{r}}\left(\tilde{r} \frac{\partial}{\partial \tilde{r}} \tilde{u}\right)+\epsilon \int_{0}^{\tilde{t}} \int_{0}^{1} \tilde{u} r^{\prime} \mathrm{d} r^{\prime} \mathrm{d} t^{\prime} \\
& +\chi\left(2 \int_{0}^{1} \tilde{u} r^{\prime} \mathrm{d} r^{\prime}-\frac{\partial}{\partial \tilde{t}} \tilde{x}_{\mathrm{p}}\right)=-(1+\gamma / \beta) \frac{\partial^{2}}{\partial \tilde{t}^{2}} \alpha \\
& \frac{\partial^{2}}{\partial \tilde{t}^{2}}\left(\tilde{x}_{\mathrm{p}}+\alpha\right)+\xi\left(\frac{\partial}{\partial \tilde{t}} \tilde{x}_{\mathrm{p}}-2 \int_{0}^{1} \tilde{u} r^{\prime} \mathrm{d} r^{\prime}\right) \\
& \quad+\frac{1}{F r^{2}} \sin \left(\tilde{x}_{p}+\alpha\right)=0
\end{aligned}
$$

with the initial and boundary conditions

$$
\begin{gathered}
\tilde{u}(\tilde{r}, 0)=\frac{\partial}{\partial \tilde{t}} \tilde{u}(\tilde{r}, 0)=\tilde{x}_{\mathrm{p}}(0)=\frac{\partial}{\partial \tilde{t}} \tilde{x}_{\mathrm{p}}(0)=0, \\
\tilde{u}(1, \tilde{t})=\frac{\partial}{\partial \tilde{r}} \tilde{u}(0, \tilde{t})=0 .
\end{gathered}
$$

In contrast to Van Buskirk's equation (2.3), the system of equations $(3.7 a)$ and $(3.7 b)$ is now nonlinear owing to the circular shape of the SCC, which leads to a sine function in the gravitational force term (3.3). The governing equations contain two new dimensionless coefficients $\xi$ and $\chi$

$$
\begin{aligned}
& \xi=\frac{9 \rho a^{2}}{2 \rho_{\mathrm{p}} a_{\mathrm{p}}^{2}}, \\
& \chi=\frac{3 a_{\mathrm{p}} n_{\mathrm{p}}}{\beta R} .
\end{aligned}
$$

It will become clear in $\$ 5.1$ that the coefficient $\xi$ determines the time it takes for particles to adjust their own velocity to the velocity of the surrounding flow. 
Table 2. Values of the dimensionless coefficients for different particle sizes $a_{\mathrm{p}}$ (the Stokes number $S t$ will be defined in $\S 5.1$ ).

\begin{tabular}{lrlrrr}
\hline$a_{\mathrm{p}}(\mu \mathrm{m})$ & $\xi$ & $\chi / n_{\mathrm{p}}$ & \multicolumn{1}{c}{ St } & $\epsilon r^{2}$ \\
\hline 1 & 42666.67 & 0.000213 & 205.58 & 0.09752 & 0.79052 \\
5 & 1706.67 & 0.001066 & 8.22 & 0.09752 & 0.79052 \\
10 & 426.67 & 0.002132 & 2.06 & 0.09752 & 0.79052 \\
15 & 189.63 & 0.003197 & 0.91 & 0.09752 & 0.79052 \\
20 & 106.67 & 0.004263 & 0.51 & 0.09752 & 0.79052 \\
25 & 68.27 & 0.005329 & 0.33 & 0.09752 & 0.79052 \\
30 & 47.41 & 0.006395 & 0.23 & 0.09752 & 0.79052 \\
\hline
\end{tabular}

The higher the value of $\xi$, the quicker the particles adjust their velocities to the surrounding flow. Typical values for $\xi$ lie around $10^{2}$ (for large particles, $a_{\mathrm{p}} \approx 20 \mu \mathrm{m}$ ) and $10^{5}$ (for very small particles, $a_{\mathrm{p}}<1 \mu \mathrm{m}$ ), which means that the particles adjust their velocity $10^{2}$ times (or $10^{5}$ times, respectively) more rapidly than $T_{\text {ref }}$. The physical meaning of the coefficient $\chi$ is much different. Its value determines the impact of the particle motion on the fluid flow. Typically, $\chi$ is very small ranging from approximately $10^{-4}$ (one very small particle) to $10^{-2}$ (many large particles). Nevertheless, it would be wrong to neglect $\chi$ since no positional nystagmus could be observed without it. Typical values for the used dimensionless coefficients are listed in table 2. Unless noted otherwise, these values will be used in $\$ 4-6$.

\section{NUMERICAL STUDY OF THE PRINCIPAL MECHANISMS}

The governing equations $(3.7 a)$ and $(3.7 b)$ can be solved numerically in a straightforward manner. The nonlinear terms are integrated with respect to time with an explicit scheme, and we use an implicit Crank-Nicolson scheme for the linear terms to cope with the numerical stiffness of the problem. The spatial derivatives are discretized with a central sixth-order compact finitedifference scheme (one-sided lower order schemes at the boundaries) on an equidistant grid (see Ferziger \& Perić 2002; Colonius \& Lele 2004). This discretization method yields an efficient and robust simulation code.

Figure 4 shows a typical solution of $(3.7 a)$ and $(3.7 b)$ for the manoeuvre from figure $3 b$. During the head manoeuvre (per-rotatory phase, $t<0$ ), we observe that the cupula displacement $V(t)$ follows qualitatively the curve of the head velocity $\dot{\alpha}(t)$. Immediately after the head manoeuvre has ended, there is an overshoot. This overshoot is somewhat smaller than that in the healthy SCC due to the influence of the particles. Up to this point, the solution is qualitatively the same as for the healthy SCC. In the post-rotatory phase $(t>0)$, the cupula displacement $V(t)$ crosses the zero axis a second time at $t=T_{\mathrm{L}}$ (onset latency) instead of relaxing to zero slowly and monotonically. The cupula returns to its relaxed position only after its deflection has reached a local extremum $-V_{\max }$ at $t=T_{\mathrm{P}}$ (time to peak). This behaviour of the cupula corresponds to the positional nystagmus observed with canalithiasis patients (cf. figure 1). The second crossing of the zero axis and the subsequent local extremum of the cupula displace-
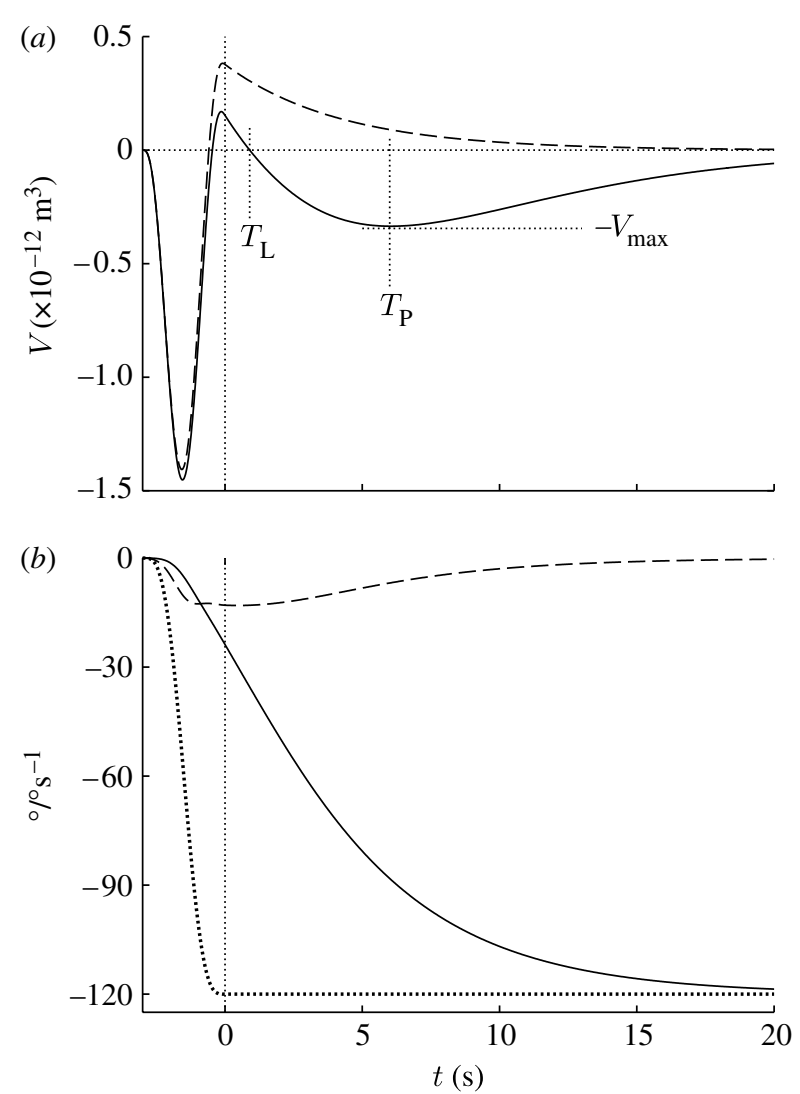

Figure 4. Numerical simulation of the (a) cupula displacement $V(t)$ (solid line, with particles; dashed line, without particles) and $(b)$ particle position $x_{\mathrm{p}}(t)$ and velocity $\dot{x}_{\mathrm{p}}(t)$ (solid line, $x_{\mathrm{p}} / R$; dashed line, $x_{\mathrm{p}} / R$; dotted line, $\left.-\alpha\right)$ for an SCC with seven particles $\left(n_{\mathrm{p}}=7\right)$ of radius $a_{\mathrm{p}}=14 \mu \mathrm{m}(\epsilon=0.09752$, $\left.\mathrm{Fr}^{2}=0.79052, \xi=217.69, \chi=0.02089\right)$. For comparison, (a) shows again the cupula displacement of a healthy SCC from figure $3 a$.

ment is perceived as a secondary angular motion that leads to the positional nystagmus and causes vertigo.

We would like to emphasize the observation that the onset latency is caused by the overshoot of the cupula. This is an alternative to the explanation offered by Squires et al. (2004), who related the onset latency to the passage of the particles from the ampulla into the slender duct. Our explanation also holds if the initial location of the particles is not in the ampulla (a possibility indicated, for instance, in appendix A of Rajguru et al. 2004).

The particle motion $x_{\mathrm{p}}(t)$ can also be split into perand post-rotatory phases. In the per-rotatory phase $(t<0)$, the particles accelerate such that they attain 
(a)
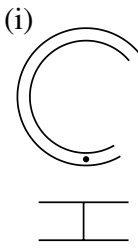

(b)

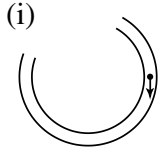

(ii)
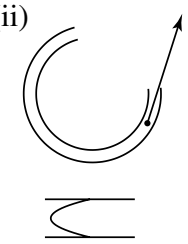

(iii)

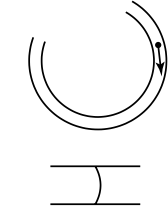

(iii)

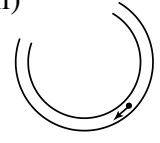

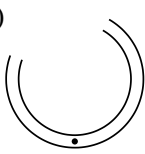

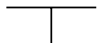

Figure 5. Schematics of the SCC with particles $\left(a_{\mathrm{p}}=11 \mu \mathrm{m}\right.$, $\left.n_{\mathrm{p}}=10 ; \epsilon=0.09752, \mathrm{Fr}^{2}=0.79052, \xi=352.61, \chi=0.02345\right)$ during a head manoeuvre. (a) Per-rotatory phase: (i) initial state, cupula relaxed $\left(\alpha=0^{\circ}, \dot{\alpha}=0^{\circ} \mathrm{s}^{-1}\right)$, (ii) maximum head velocity, cupula fully deflected $\left(\alpha=60^{\circ}, \dot{\alpha}=87.5^{\circ} \mathrm{s}^{-1}\right)$ and (iii) end of head manoeuvre, cupula overshoot $\left(\alpha=120^{\circ}\right.$, $\left.\dot{\alpha}=0^{\circ} \mathrm{s}^{-1}\right)$. (b) Post-rotatory phase: (i) end of latency period, $t=T_{\mathrm{L}}\left(\alpha=120^{\circ}, \dot{\alpha}=0^{\circ} \mathrm{s}^{-1}\right)$, (ii) peak nystagmus, $t=T_{\mathrm{P}}$ $\left(\alpha=120^{\circ}, \dot{\alpha}=0^{\circ} \mathrm{s}^{-1}\right)$ and (iii) return to relaxed state, $t \rightarrow \infty$ $\left(\alpha=120^{\circ}, \dot{\alpha}=0^{\circ} \mathrm{s}^{-1}\right)$ (the arrow shows the absolute particle velocity $\dot{x}_{\mathrm{p}}+R \dot{\alpha}$; the cupula is drawn separately below the canal as a bulged membrane).

their maximum velocity at the end of the head manoeuvre. During the post-rotatory phase $(t>0)$, the particles fall down through the canal. At the same time, they gradually decelerate such that they come to a halt when they reach the lowest position in the canal. There is no oscillation about the lowest position. The particles behave like the mass of an over-damped pendulum.

The corresponding results for $n_{\mathrm{p}}=10$ and $a_{\mathrm{p}}=11 \mu \mathrm{m}$ are shown in figure 5 as a sequence of schematics. It documents the two-phase process of canalithiasis: particle positioning during the per-rotatory phase and secondary endolymph flow during the post-rotatory phase, which is induced by the falling particles.

We study the influence of $n_{\mathrm{p}}$ and $a_{\mathrm{p}}$ on the characteristic values $T_{\mathrm{L}}, T_{\mathrm{P}}$ and $V_{\max }$ by varying $n_{\mathrm{p}}$ from 1 to 50 and $a_{\mathrm{p}}$ from 1 to $30 \mu \mathrm{m}$ (figure 6 ). Note that we are covering here a wider range of particle sizes than that suggested by Campos (1990) because we also want to consider large lumps of smaller particles. As mentioned earlier, the actual number and size of particles present in the SCC of a canalithiasis patient is not known. We can only estimate the particle size and the number by comparing clinical data with the results from theoretical models and experiments. Rajguru \& Rabbitt (2007) concluded from their study that canalithiasis 'most likely involves particles of the order of $5-20 \mu \mathrm{m}$ '. We will try in $\$ 5.2$ to estimate the particle size for the data shown in figure 1.

As a general trend, we observe that more and larger particles lead to a shorter onset latency and an earlier and more intense peak nystagmus. Curve fitting suggests that the magnitude of the positional nystagmus is proportional to the total particle cross section $\left(\pi a_{\mathrm{p}}^{2} n_{\mathrm{p}}\right)$. There are also configurations for which the onset latency $T_{\mathrm{L}}$ vanishes (figure $7 a$ ). In these cases, the
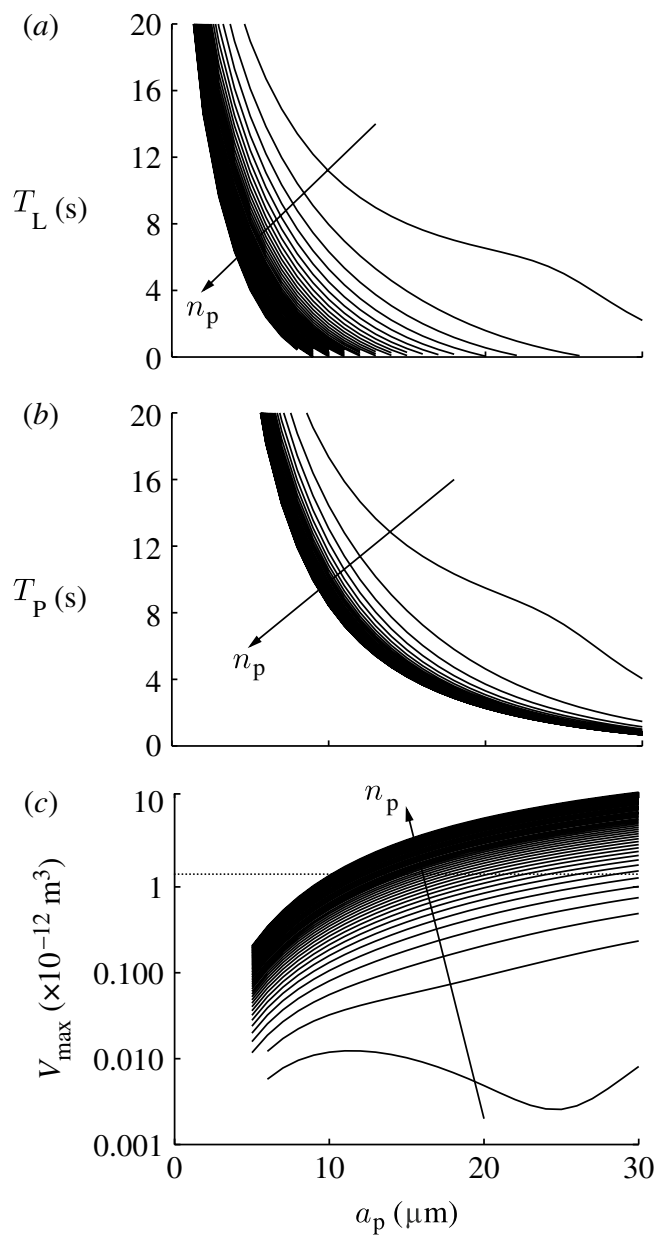

Figure 6. Influence of the particle size $a_{\mathrm{p}}$ and the particle number $n_{\mathrm{p}}$ on the characteristic values of the positional nystagmus (curves for $n_{\mathrm{p}}=1,2, \ldots, 50$ ); the dotted line in $(c$ ) indicates the maximum cupula displacement in the perrotatory phase.

particle-induced flow is so strong that there is no overshoot, i.e. the positional nystagmus follows the head movement seamlessly (figure $7 b$ ). This phenomenon has also been observed in clinical experiments by the second author.

Rajguru et al. (2004) found that the time to peak is proportional to $1 / a_{\mathrm{p}}^{2}$ if the total particle mass $M_{\mathrm{p}}=n_{\mathrm{p}} m_{\mathrm{p}}$ is kept constant (figure $5 c$ in their paper). Our results (figure $8 a$ ) agree with their observations at least for smaller particles and large $M_{\mathrm{p}}$. Furthermore, in figure $8 b$, we observe that the peak nystagmus decreases for larger particles if $M_{\mathrm{p}}$ is kept constant. To understand this effect, we need to take into account our previous observation that $V_{\max }$ scales approximately like $a_{\mathrm{p}}^{2} n_{\mathrm{p}}$, and at the same time we keep the total particle mass constant, i.e. $n_{\mathrm{p}} \propto a_{\mathrm{p}}^{-3}$ constant. It follows that $V_{\max }$ must decrease with increasing particle size.

\subsection{Two-dimensional particle model}

So far, we have used only the one-dimensional particle model first proposed by House \& Honrubia (2003). However, it is not clear a priori whether this is indeed an appropriate model, whether we might be missing 

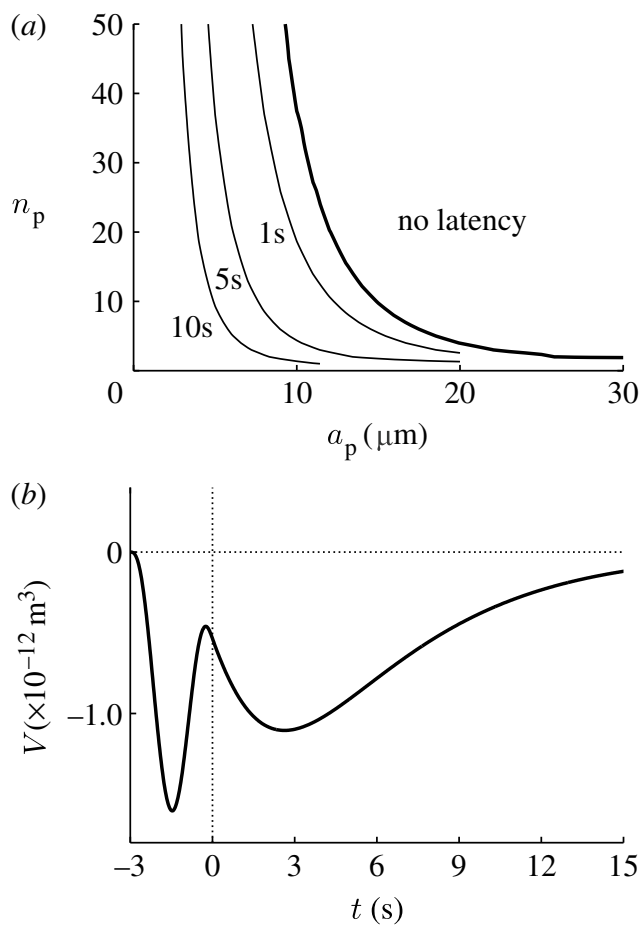

Figure 7. Vanishing onset latency. (a) Latency $T_{\mathrm{L}}$ as a function of the particle size $a_{\mathrm{p}}$ and number $n_{\mathrm{p}}$ and $(b)$ cupula displacement $V(t)$ for a configuration without onset latency $\left(a_{\mathrm{p}}=20 \mu \mathrm{m}, n_{\mathrm{p}}=10\right)$.

some fundamental effects or, even worse, whether this model yields misleading results. Therefore, we compared our results with those obtained from the two-dimensional particle model by Squires et al.

To this end, we added an equation for the radial particle motion to the governing equations $(3.7 a)$ and $(3.7 b)$. The drag forces in both particle equations were adjusted to reflect the influence of the pipe walls (Bungay \& Brenner 1973; Happel \& Brenner 1973; Squires et al. 2004). We modified the pressure drop due to the moving particles according to Squires et al. Details can be found in the appendix of Squires et al. (2004) and references therein. To be consistent with Squires et al. we also introduced a lubrication gap of $1 \mu \mathrm{m}$. In addition, we included a centrifugal force $m_{\mathrm{p}} R$ $\dot{\alpha}^{2}$ to reflect the influence of the rotating reference frame. The resulting equations for the two-dimensional model were solved within the simulation framework described earlier.

Figure 9 shows the particle trajectories obtained with the two-dimensional model during and after the head manoeuvre for different particle sizes. During most of the head manoeuvre, the particles remain in contact (apart from the lubrication gap) with the outer wall. Smaller particles remain at the wall even beyond $90^{\circ}$ due to the centrifugal forces. Only when the head manoeuvre comes to an end, the particles start falling down in a more or less straight line until they hit either the inner or outer wall of the SCC. The very small particles touch the inner wall first and detach a second time before they hit the outer wall. The situation is different for large particles. During the head manoeuvre, they slide down along the wall so much that they never detach from the wall. The one-dimensional model
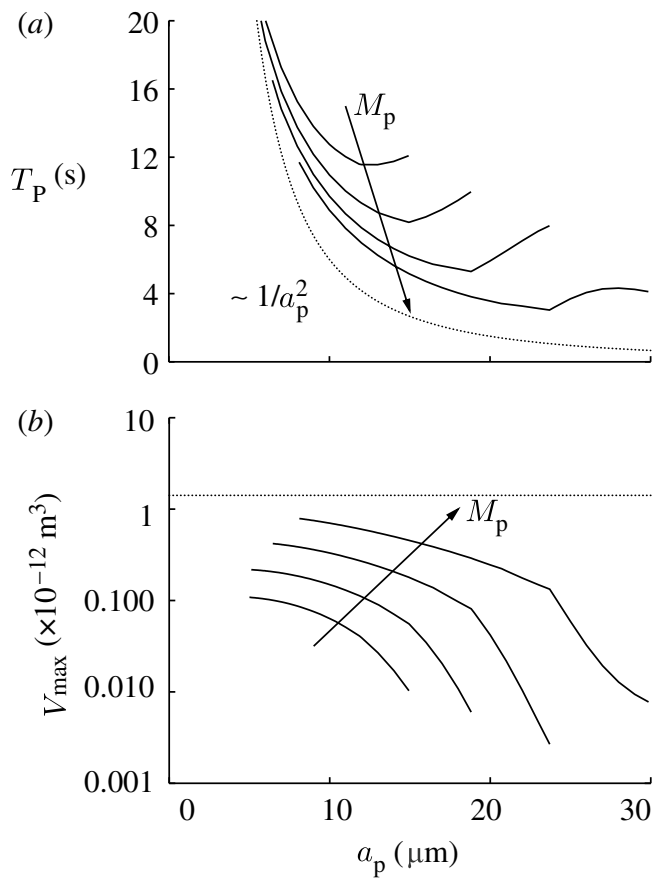

Figure 8. (a) Time to peak $T_{\mathrm{P}}$ and (b) cupula displacement $V_{\max }$ as a function of $a_{\mathrm{p}}$ if the total particle mass $M_{\mathrm{p}}$ is kept constant $\left(n_{\mathrm{p}} \propto a_{\mathrm{p}}^{-3}\right)$. The dotted line in $(a)$ indicates the proportionality of $T_{\mathrm{P}}$ to $1 / a_{\mathrm{p}}^{2}$, and the dotted line in $(b)$ indicates the maximum cupula displacement in the perrotatory phase.
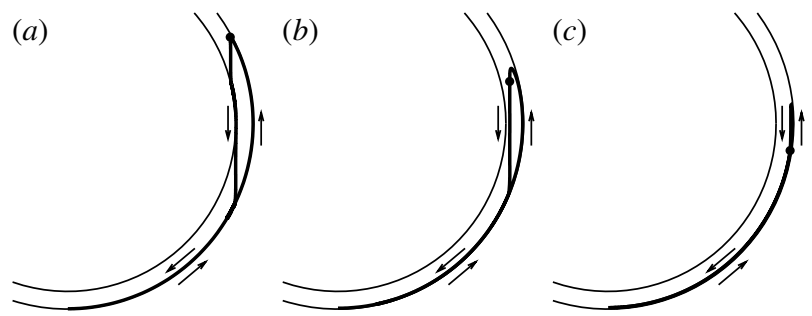

Figure 9. Particle trajectories for $n_{\mathrm{p}}=5$ and $(a) a_{\mathrm{p}}=5 \mu \mathrm{m}$, (b) $15 \mu \mathrm{m}$ and (c) $25 \mu \mathrm{m}$ (dot indicates the location of the particles at the end of the head manoeuvre).

neglects these effects. Nevertheless, we will see in the following that these effects are not central to the principal mechanisms of canalithiasis.

Figure 10 compares the axial particle velocities for the three cases from figure 9 . The rapid changes in the axial velocities in the two-dimensional model correspond to the instants when the particles either hit or detach from the wall. In general, the axial particle velocity is smaller with the two-dimensional model. However, although the two-dimensional model reveals more detail in the particle dynamics, the two models describe the same principal behaviour and give similar results.

Finally, we compare the cupula displacements, the key indicator for the perceived angular velocity (figure 11). Again, the results differ somewhat quantitatively for the two particle models, but the basic character of the results remains the same: a trend towards shorter onset latency and stronger nystagmus for larger particles, no latency for very large particles, etc. Apparently, we are not missing any basic feature of the positional nystagmus if we use the one-dimensional 

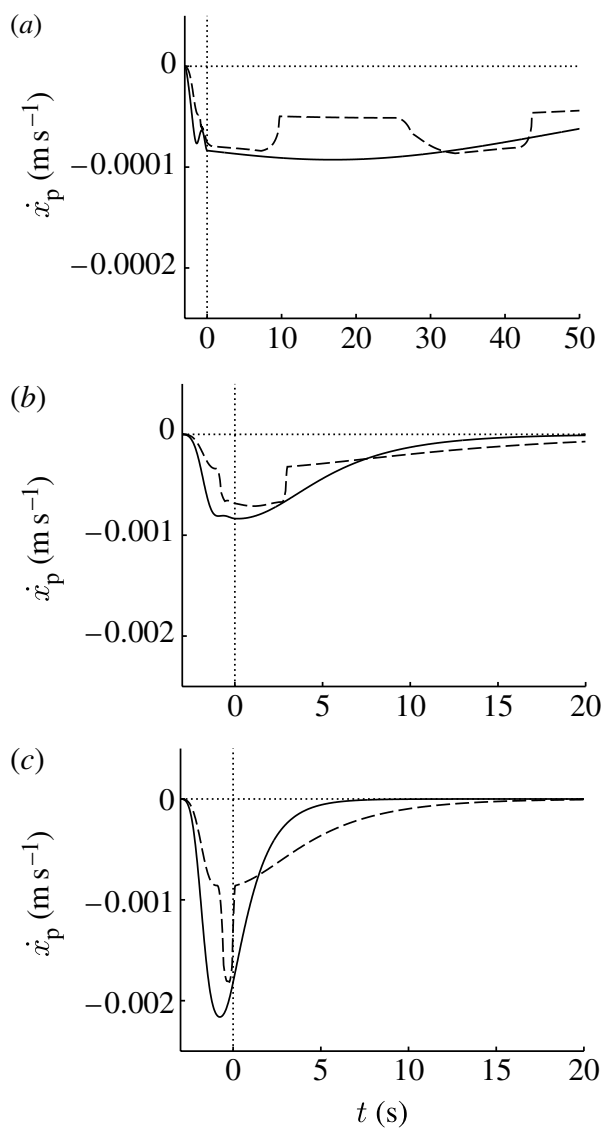

Figure 10. Axial particle velocity $\dot{x}_{\mathrm{p}}(t)$ for $n_{\mathrm{p}}=5$ and $(a) a_{\mathrm{p}}=$ $5 \mu \mathrm{m},(b) 15 \mu \mathrm{m}$ and (c) $25 \mu \mathrm{m}$ (solid line, one-dimensional particle model; dashed line, two-dimensional particle model; note the different ranges for $(a)$ ).

model. We conclude that the two different particle models yield (in the spirit of a first approximation) equivalent results. The following observations can help explain this somewhat surprising fact.

(i) The smaller particles spend a certain amount of time close to the centreline of the canal. During this time (coincidental with the build-up of the positional nystagmus), the pressure drop in the two-dimensional model is larger than that in the one-dimensional model. While the particles are closer to the wall the situation is reversed. On average, the two effects tend to cancel.

(ii) Owing to their size (relative to the canal), the large particles induce a pressure drop that is large enough to cause a positional nystagmus even while they are only sliding along the wall. This is consistent with the experiment of Rajguru \& Rabbitt (2007). It has to be noted, however, that our results are very sensitive to the choice of the lubrication gap. This point requires further investigation.

(iii) Mathematically speaking the cupula has the role of a temporal integrator which tends to smooth differences in the particle dynamics.

The two-dimensional particle model adds an additional level of detail to the results, which is valuable for the
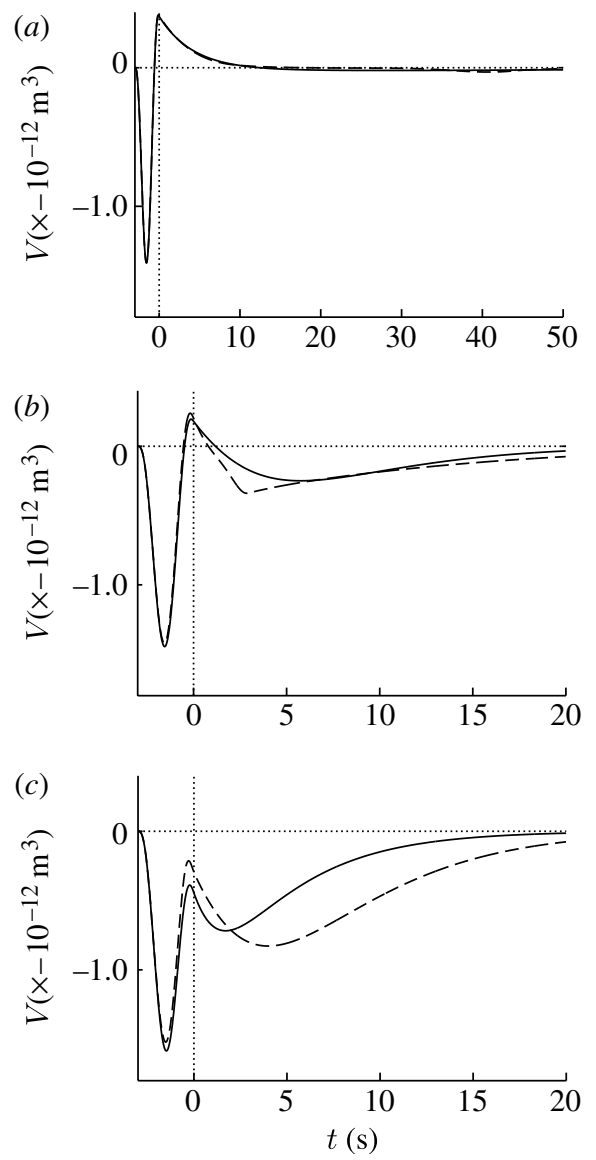

Figure 11. Cupula displacement $V(t)$ for $n_{\mathrm{p}}=5$ and $(a) a_{\mathrm{p}}=$ $5 \mu \mathrm{m},(b) 15 \mu \mathrm{m}$ and (c) $25 \mu \mathrm{m}$ (solid line, one-dimensional particle model; dashed line, two-dimensional particle model).

study of the more intricate features of canalithiasis. Nevertheless, the one-dimensional model appears to be sufficient for the study of the principal features of canalithiasis, and in the following we will continue our study with this model.

\section{ANALYSIS OF THE LINEARIZED EQUATIONS}

In order to obtain a deeper understanding of the fluid-particle dynamics in the SCC, we linearize the governing equations $(3.7 a)$ and $(3.7 b)$. To this end, we assume that the angle $\phi=x_{\mathrm{p}} / R+\alpha$ (spanned by the particle angle $x_{\mathrm{p}} / R$ and the direction of gravity $-\alpha$ ) is small, such that

$$
\sin \phi \approx \phi=x_{\mathrm{p}} / R+\alpha .
$$

This approximation may introduce a sizable error for angles of $90^{\circ}$ and more, but we will see that the results obtained from the linearized problem remain qualitatively correct and that they will help us to understand the observations of $\S 4$.

We write the linearized governing equations $(3.7 a)$ and $(3.7 b)$ as

$$
\frac{\partial}{\partial \tilde{t}} \boldsymbol{Q u}=\boldsymbol{P u}+\boldsymbol{f}
$$

where $\boldsymbol{Q}$ and $\boldsymbol{P}$ are operator matrices. The vector $\boldsymbol{u}$ contains the dependent variables $\tilde{u}$ and $\tilde{x}_{\mathrm{p}}$ and $\boldsymbol{f}$ contains the forcing terms. 

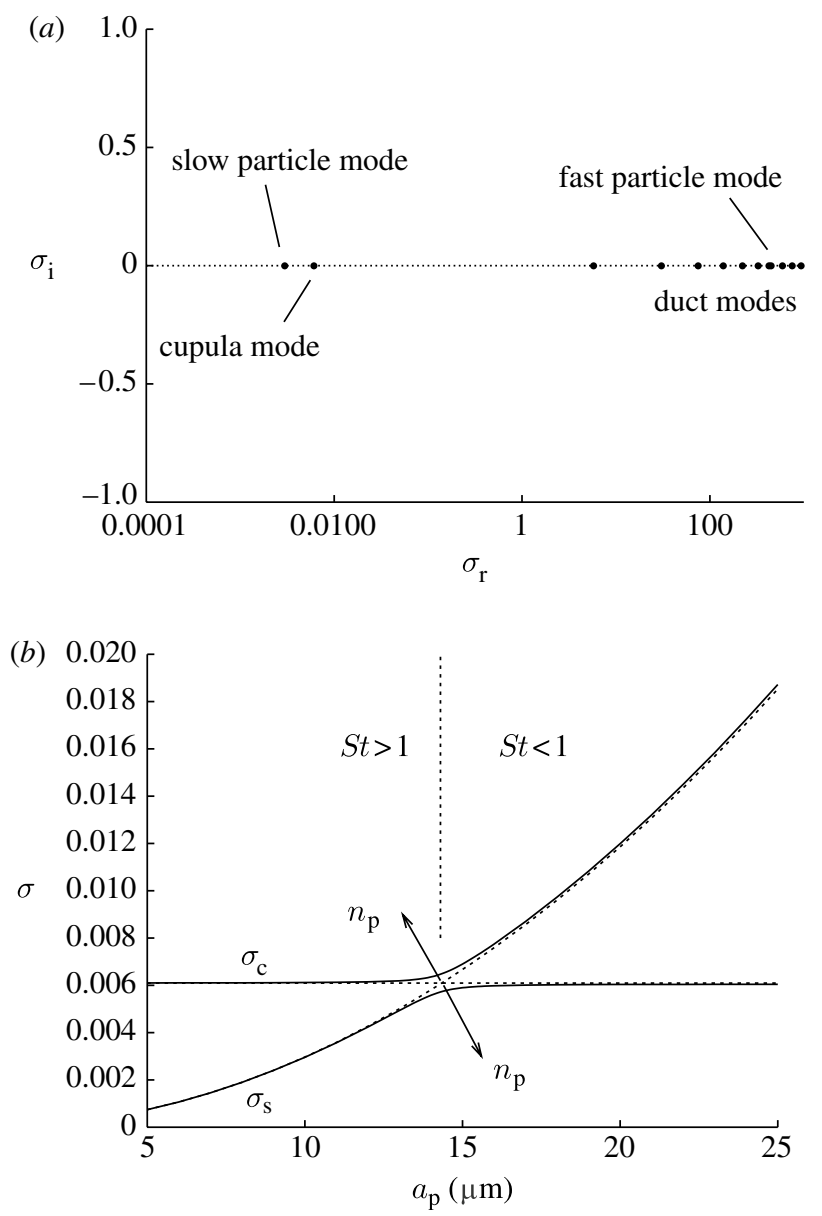

Figure 12. (a) Eigenvalues $\sigma=\sigma_{\mathrm{r}}+\mathrm{i} \sigma_{\mathrm{i}}$ for 10 particles with radius $a_{\mathrm{p}}=10 \mu \mathrm{m}$. (b) Eigenvalues $\sigma_{\mathrm{s}}$ (slow particle mode) and $\sigma_{\mathrm{c}}$ (cupula mode) as a function of the particle radius $a_{\mathrm{p}}$ $\left(n_{\mathrm{p}}=10\right)$. The dashed lines show the estimates $\epsilon / 16$ and $1 /\left(F^{2} \xi\right)$.

\subsection{Temporal spectrum}

To study the temporal spectrum of (5.1), we set $\dot{\alpha}$ to zero, which corresponds to the situation after the head manoeuvre $(t>0 \mathrm{~s})$. The governing equations (5.1) then form a homogeneous initial-value problem in which the initial values are the result of a completed head manoeuvre. With the separation ansatz

$$
\boldsymbol{u}(\tilde{r}, \tilde{t})=\hat{\boldsymbol{u}}(\tilde{r}) \mathrm{e}^{-\sigma \tilde{t}}
$$

we obtain the generalized eigenvalue problem

$$
-\sigma \boldsymbol{Q} \hat{\boldsymbol{u}}=\boldsymbol{P} \hat{\boldsymbol{u}}
$$

A numerical solution of this problem is shown in figure $12 a$. The eigenvalues of the cupular mode and the duct modes are nearly at the same locations as in the configuration without particles (cf. $\$ 2$ and fig. 3 in Obrist 2008). In addition, there are two new eigenvalues attributable to the particle equation. We can find estimates for the two new eigenvalues by analysing the decoupled particle equation

$$
\frac{\partial^{2}}{\partial \tilde{t}^{2}} \tilde{x}_{\mathrm{p}}+\xi \frac{\partial}{\partial \tilde{t}} \tilde{x}_{\mathrm{p}}+F r^{-2} \tilde{x}_{\mathrm{p}}=0 .
$$

Assuming that $\mathrm{Fr}^{-2} \ll \xi$ we find

$$
\sigma_{\mathrm{s}} \approx \frac{1}{F r^{2} \xi}, \quad \sigma_{\mathrm{f}} \approx \xi .
$$

We name the corresponding modes the slow particle mode $\sigma_{\mathrm{s}}$ and the fast particle mode $\sigma_{\mathrm{f}}$. Inspection of the numerical results shows that the expressions (5.5) are indeed good estimates for the eigenvalues of (5.3).

These results motivate the definition of a Stokes number that relates a typical time constant for the particle motion to a typical time constant of the fluid flow. The classical choice for a Stokes number relates the fast particle mode to the viscous time scale $T_{\text {ref }}$

$$
S t_{\text {classical }}=\xi^{-1} \ll 1 .
$$

The fact that $S t_{\text {classical }}$ is very small for typical particle sizes indicates that the particles behave like passive tracers that follow the streamlines and have negligible influence on the fluid (e.g. Clift et al. 1978). Obviously, this Stokes number does not tell the full story and it is not useful in the context of canalithiasis. Therefore, we discard the definition (5.6) and define a different Stokes number from the estimated time scales for the slow particle mode and the cupula mode

$$
S t=\frac{\sigma_{\mathrm{c}}}{\sigma_{\mathrm{s}}}=\frac{\epsilon F r^{2} \xi}{16}=\frac{9 \pi K a^{4}}{16 \beta a_{\mathrm{p}}^{2} g\left(\rho_{\mathrm{p}}-\rho\right)} .
$$

For the typical parameters given in table 1, we find that St may be below as well as above unity. We will see in the following that this Stokes number and the two least stable modes (cupula and slow particle modes) play a central role in canalithiasis.

Therefore, we examine these two modes in some more detail. The two estimates (2.5) and (5.5) actually suggest that the two eigenvalues coincide for $S t=1$. For the standard parameters from table 1 , this would be the case for $a_{\mathrm{p}} \approx 14.3 \mu \mathrm{m}$. Figure $12 b$ investigates this idea by comparing the approximate expressions (2.5) and (5.5) with the numerically computed eigenvalues. We find that the two eigenvalues never coincide. Rather, they repel each other as we approach $S t=1$. This repulsion becomes stronger for larger numbers of particles $n_{\mathrm{p}}$. For small particle radii $(S t>1)$, the eigenvalues follow closely the estimates (2.5) and (5.5). For $S t<1$, however, the eigenvalues have switched roles: the cupula eigenvalue $\sigma_{\mathrm{c}}$ follows the estimated curve for the slow particle mode and vice versa. As a result, the cupula mode always decays faster than the slow particle mode. In other words, the slow particle mode will prevail as $t \rightarrow \infty$.

The change of roles shows also in figure $13 a$ where we plotted the ratio $\theta$ between the bulk velocity

$$
\bar{u}=2 \int_{0}^{1} u r \mathrm{~d} r
$$

and the particle velocity $-\sigma \hat{x}_{\mathrm{p}}$, where $\theta_{\mathrm{c}}$ and $\theta_{\mathrm{s}}$ are the ratios for the cupula and slow particle modes, respectively. This plot shows us two things. First, we note that the fluid and particle velocities always point in the same direction (co-flow) in the cupula mode, whereas they always point in opposite directions (counter-flow) in 

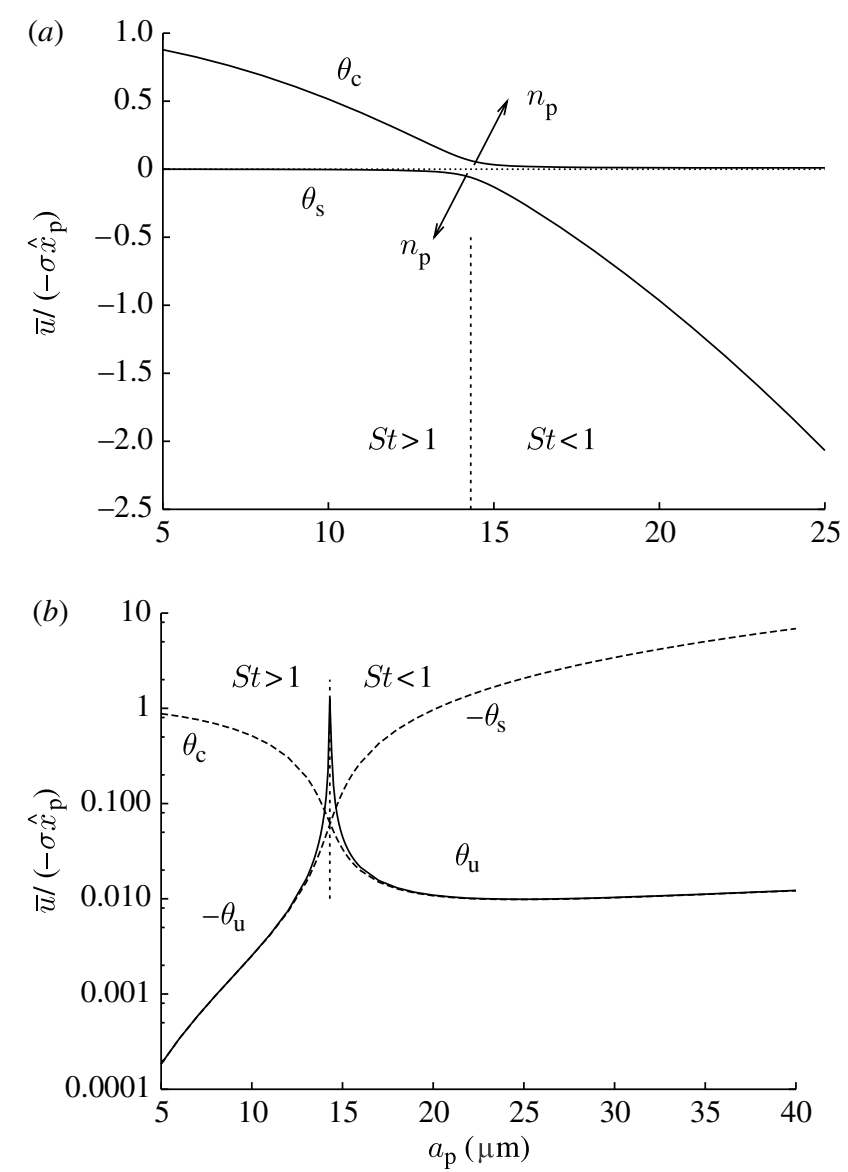

Figure 13. (a) Ratio $\theta$ between the average fluid velocity $\bar{u}$ and the particle velocity $-\sigma \hat{x}_{\mathrm{p}}$ of the cupula and slow particle modes as a function of the particle radius $a_{\mathrm{p}}\left(n_{\mathrm{p}}=10\right)$. (b) The same data on a logarithmic scale together with the approximate expression $\theta_{\mathrm{u}}$.

the slow particle mode. Second, we find that the particle velocity is dominant $\left(|\bar{u}| \ll\left|-\sigma \hat{x}_{\mathrm{p}}\right|\right)$ in the slow particle mode for $S t>1$ and that it is dominant in the cupula mode for $S t<1$. Hence, the switching of roles at $S t=1$ is manifested in the dominance of the particle velocity over the fluid velocity.

As a final result in this section, we derive the universal approximation $\theta_{\mathrm{u}}$. To this end, we enter the approximate eigensolution for the slow particle mode into the linearized governing equations, i.e. a parabolic velocity profile for the endolymph and $\hat{x} \exp \left(-\tilde{t} / F r^{2} \xi\right)$ for the particle motion. From this, we obtain

$$
\theta_{\mathrm{u}}=\frac{\bar{u}}{-\sigma \hat{x}}=\frac{-\chi S t}{16 S t^{2}-(16+2 \chi) S t+\epsilon / 4} .
$$

This expression approximates $\theta_{\mathrm{c}}$ for $S t<1$ and $\theta_{\mathrm{s}}$ for St $>1$ (figure 13b).

We would like to emphasize that the number of particles $n_{\mathrm{p}}$ plays a small but, nevertheless, very important role here. Figure $12 b$ shows that the values of $\sigma_{\mathrm{c}}$ and $\sigma_{\mathrm{s}}$ can be predicted relatively well by the estimates (2.5) and (5.5), which are both independent of the particle number. Only for $S t \approx 1$ the particle number has a noticeable influence on $\sigma_{\mathrm{s}}$ and $\sigma_{\mathrm{c}}$. In that sense, the influence of $n_{\mathrm{p}}$ is small. At the same time, the effect of a non-zero $n_{\mathrm{p}}$ is huge in the sense that the cupula and fast particle modes switch roles at $S t=1$. They would not do that if $n_{\mathrm{p}}$ were zero. This observation goes along with our comment at the end of $\S 3$, where we mentioned that $\chi$ (which is proportional to $n_{\mathrm{p}}$ ) must not be neglected, even though it is very small.

\subsection{Reduced-order model}

Figures 1 and 4 suggest that the cupula oscillates about its relaxed state at least once during the first few seconds after the head manoeuvre. This appears to contradict the results from $\$ 5.1$ where we have found that all modes decay monotonically as $t \rightarrow \infty$. To explain this transient oscillation, we reduce our dynamical system to the two least stable modes. We can do this because all energy in the other modes dissipates faster than $T_{\text {ref }}$ after the head manoeuvre has ended. Therefore, these higher modes are irrelevant to the slow dynamics of the positional nystagmus. (They are very relevant, however, during the per-rotatory phase, as shown by Obrist (2008).)

To formulate the reduced-order model, we reduce the flow profile $\tilde{u}(\tilde{r}, \tilde{t})$ to the bulk velocity $\bar{u}(\tilde{t})$. Likewise, the $k$ th eigenfunction $\left(\hat{u}_{k}(\tilde{r}),-\sigma_{k} \hat{x}_{\mathrm{p}, k}\right)$ reduces to the vector $\left(\bar{u}_{k},-\sigma_{k} \hat{x}_{\mathrm{p}, k}\right)$. This reduced system has the general solution

$\left(\begin{array}{c}\bar{u} \\ \partial \tilde{x}_{\mathrm{p}} / \partial \tilde{t}\end{array}\right)=A_{1}\left(\begin{array}{c}\bar{u}_{\mathrm{s}} \\ -\sigma_{\mathrm{s}} \hat{x}_{\mathrm{p}, \mathrm{s}}\end{array}\right) \mathrm{e}^{-\sigma_{\mathrm{s}} \tilde{t}}+A_{2}\left(\begin{array}{c}\bar{u}_{\mathrm{c}} \\ -\sigma_{\mathrm{c}} \hat{x}_{\mathrm{p}, \mathrm{c}}\end{array}\right) \mathrm{e}^{-\sigma_{\mathrm{c}} \tilde{t}}$.

If we know the initial conditions, i.e. the fluid velocity $\bar{u}_{0}$ and the particle velocity $\tilde{x}_{\mathrm{p}, 0}^{(1)}$ immediately after the head manoeuvre $(\tilde{t}=0)$, we can determine the constants $A_{1,2}$.

It can be shown (see appendix B for more detail) that for appropriate $A_{1,2}$ the reduced-order model (5.10) exhibits exactly the transient oscillations of the cupula, which we observed in figures 1 and 4 . Furthermore, the reduced-order model can be used to derive explicit relationships between the dimensionless coefficients and the characteristic values of the positional nystagmus (see also appendix B). One of these relationships stands out since it is relatively simple and might help to estimate the particle size directly from clinical data. This relationship describes the time between the onset latency and the peak nystagmus as

$$
T_{\mathrm{P}}-T_{\mathrm{L}}=\frac{16}{\epsilon} \frac{S t}{S t-1} \ln S t .
$$

It shows us that $T_{\mathrm{P}}-T_{\mathrm{L}}$ depends strongly on the Stokes number and that it is independent of the number of particles (keep in mind here that this relationship is only an approximation). We have used this relationship to plot $T_{\mathrm{P}}-T_{\mathrm{L}}$ as a function of the particle size (figure 14). A comparison of estimates from (5.11) with numerical solutions of the full nonlinear governing equations (3.7a) and (3.7b) shows that figure 14 slightly underestimates the particle size. Nevertheless, figure 14 can serve as a valuable tool for diagnosing the particle size. The patient in figure 1 , for example, exhibits a difference between $T_{\mathrm{P}}$ and $T_{\mathrm{L}}$ of approximately $8 \mathrm{~s}$. With the help of figure 14, we can now estimate a particle size of $6 \mu \mathrm{m}$. 


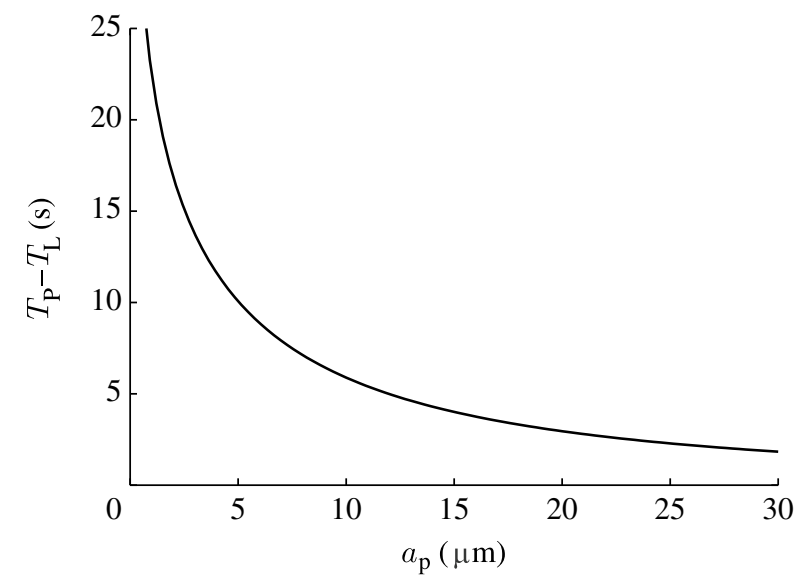

Figure 14. Estimate for the difference between time to peak $T_{\mathrm{P}}$ and onset latency $T_{\mathrm{L}}$ as a function of the particle size according to the relationship (5.11) $\left(\epsilon=0.09752, \mathrm{Fr}^{2}=0.79052\right)$.

\section{CONCLUDING REMARKS}

We have proposed and analysed an analytical model for canalithiasis, which describes the fluid-particle dynamics on the basis of the first principles. It consists of the Navier-Stokes equations for axial duct flow and an equation of motion for the particles based on Newton's second law. The two equations are coupled through the Stokes drag of the sedimenting particles. In formulating the model, we have aimed at reducing the physics of canalithiasis as much as possible. The proposed model is simple but it reproduces the characteristic features of canalithiasis (onset latency, rapid increase to peak nystagmus and a subsequent slow decay of the positional nystagmus). We demonstrated in $\$ 4.1$ with a brief numerical study that the one-dimensional particle model that neglects the radial position of the particles is sufficiently accurate to investigate the principal mechanisms.

We presented numerical and analytical evidence that variations in the cross-sectional area are not necessary conditions for a positional nystagmus. We showed that the onset latency is closely related to the overshoot of the cupula, and we demonstrated cases where there is no latency period at all (figure 7). A numerical parameter study demonstrated the general trend towards shorter onset latency and an earlier and more intense peak nystagmus for larger and more particles. We found that the intensity of the nystagmus is approximately proportional to the total particle cross section, i.e. the cross section of a single particle times the number of particles. For the special case of a constant total particle mass, we showed that the positional nystagmus is stronger if the mass is distributed to many small particles rather than few large particles (figure 8). Finally, a reduced-order model for the post-rotatory phase explained the transient mechanisms leading to a positional nystagmus. It also allowed us to derive explicit relationships between the phenomena of canalithiasis and the physical properties of the fluid, the SCC and the particles.

We showed in $\$ 5.2$ that the results may be used to estimate the size of the particles from the results of a clinical experiment. Nevertheless, it is not possible to determine the particle size and number conclusively from our model. We need to keep in mind that our model does not consider, for example, non-spherical particles or even multiple particles of different sizes. Our choice of 10 particles for most of our numerical experiments was arbitrary. At the current stage of abstraction and idealization, our model is capable of revealing the fundamental mechanisms and the basic relationships between particle size and number and the positional nystagmus.

However, the simplicity of our model with four dimensionless numbers (cupula stiffness coefficient $\epsilon$ (2.4), Froude number $\operatorname{Fr}(3.6)$, Stokes number $S t$ (5.7) and coupling coefficient $\chi(3.9 b))$ offers the possibility of studying the influence of a wide range of configurations. In the present work, we have limited our investigation to variations of $S t$ and $\chi$ (particle size and number). It appears promising at this point to further investigate the influence of $\epsilon$ and $\operatorname{Fr}$ (e.g. the cupula stiffness and the canal dimension), which might reveal other intriguing phenomena of the fluid-particle dynamics in the SCC, e.g. the different typical onset latencies for the posterior and lateral SCC.

The clinical experiment adheres to the principles of the Declaration of Helsinki. It was approved by the local ethics committee. The patient gave her written informed consent after the experimental procedure had been explained.

The authors thank L. Kleiser for many helpful discussions on this research topic. They would also like to thank C. Bockisch for providing support for the clinical experiments (e.g. figure 1).

\section{APPENDIX A. DERIVATION OF VAN BUSKIRK'S EQUATION FOR THE ENDOLYMPH FLOW IN THE SLENDER PART OF A SEMICIRCULAR CANAL}

In the following, we give a short summary of the derivation of Van Buskirk's equation for the endolymph flow in the slender part of an SCC. For more detailed information and solutions to this equation, the reader is referred to Van Buskirk et al. (1976) and Obrist (2008).

An observer moving with the SCC (figure 2) perceives a flow of the endolymph with an axial velocity $u$. This fluid motion obeys the Navier-Stokes equations that consist of an equation for the conservation of mass and three equations for the conservation of momentum. In the slender part of the SCC, the equation for the conservation of the axial momentum takes the simple form

$$
\frac{\partial(u+R \dot{\alpha})}{\partial t}=-\frac{1}{\rho} \frac{\partial p}{\partial x}+\frac{\nu}{r} \frac{\partial}{\partial r}\left[r \frac{\partial u}{\partial r}\right],
$$

where we have neglected the influence of curvature since $R \gg a$. In this equation $\dot{\alpha}(t)$ is the angular velocity of the SCC, $\rho$ is the fluid density, $p$ is the fluid pressure and $\nu$ is the kinematic viscosity. The variables $r$ and $x$ are components of a cylindrical coordinate system with its origin on the canal centreline. Note that this equation is linear. The nonlinear terms that are usually present in 
the Navier-Stokes equations are identical to zero owing to the axial symmetry of the flow field and the continuity equation that requires that the axial velocity $u$ in the slender part of the SCC is constant in $x$. Therefore, $u$ is only a function of $r$ and $t$.

We differentiate the axial momentum equation once with respect to $x$ to find an expression for the pressure gradient. All terms with $u$ and $\dot{\alpha}$ drop out since they are constant in $x$ and we obtain

$$
\frac{\partial^{2} p}{\partial x^{2}}=0
$$

Therefore, the pressure $p$ is a linear function of $x$ and the pressure difference between the two ends of the slender part of the SCC can be written as

$$
p(\beta R)-p(0)=\beta R\left(\frac{\partial p}{\partial x}\right) .
$$

This pressure difference can be regarded as the result of an external force $F$ that exerts a pressure $F /\left(\pi a^{2}\right)$ on one end of the SCC. This gives

$$
\frac{\partial p}{\partial x}=\frac{F}{\pi a^{2} \beta R} .
$$

The external force $F$ consists of the reactive force $F_{\mathrm{c}}$ of the deflected cupula and of the inertial force $F_{\mathrm{i}}$ of the fluid in the utricle. The viscous forces of the fluid in the utricle can be neglected owing to the small flow velocities (Van Buskirk 1977). The reactive force $F_{\mathrm{c}}$ of the cupula is assumed to be proportional to the volume $V$ under the deflected cupula

$$
\frac{F_{\mathrm{c}}}{\pi a^{2}}=K \cdot V=K 2 \pi \int_{0}^{t} \int_{0}^{a} u\left(r^{\prime}, \tau\right) r^{\prime} \mathrm{d} r^{\prime} \mathrm{d} \tau,
$$

where $K$ determines the mechanical stiffness of the cupula. More sophisticated mechanical models for the cupula were studied by Rabbitt \& Damiano (1992) and Yamauchi et al. (2001). According to Newton's second law, the inertial force $F_{\mathrm{i}}$ of the fluid in the utricle is $F_{\mathrm{i}}=m_{\mathrm{u}} R \ddot{\alpha}$. The mass $m_{\mathrm{u}}$ is equal to the mass of the endolymph that is contained in a torus section of length $\gamma R$ (arc length of the utricle) with cross section $\pi a^{2}$ (cross section of the slender canal),

$$
F_{\mathrm{i}}=\rho \pi a^{2} \gamma R^{2} \ddot{\alpha} .
$$

Of course, the remainder of the fluid in the utricle also leads to an inertial force. However, this force is equilibrated by the utricle walls and it is not relevant to the flow in the slender part of the SCC.

With these expressions, we obtain the equation for the endolymph flow in the slender part of the SCC proposed by Van Buskirk et al. (1976)

$$
\frac{\partial u}{\partial t}+\left(1+\frac{\gamma}{\beta}\right) R \ddot{\alpha}=-\frac{2 \pi K}{\rho \beta R} \int_{0}^{t} \int_{0}^{a} u r^{\prime} \mathrm{d} r^{\prime} \mathrm{d} \tau+\frac{\nu}{r} \frac{\partial}{\partial r}\left[r \frac{\partial u}{\partial r}\right] .
$$

\section{APPENDIX B. EXPLICIT EXPRESSIONS FOR THE ONSET LATENCY, TIME TO PEAK AND MAXIMUM CUPULA DEFLECTION}

Starting from the reduced-order model (5.10), we derive explicit relationships for the onset latency $T_{\mathrm{L}}$, the time to peak $T_{\mathrm{P}}$ and the maximum cupula deflection $V_{\max }$. (a)

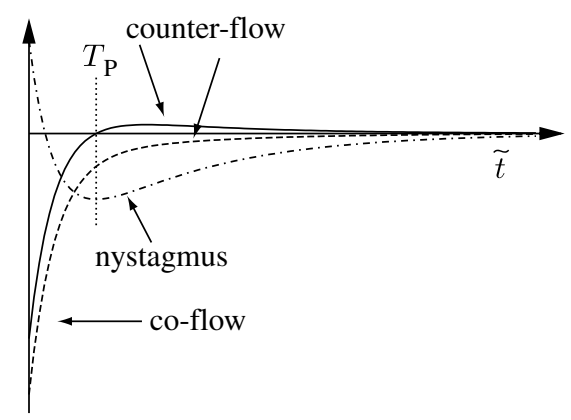

(b)

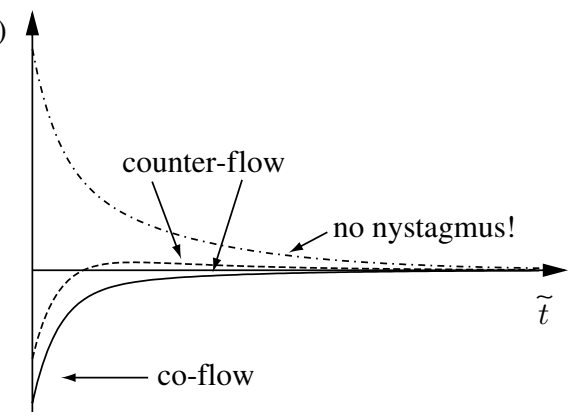

Figure 15. Two possible cases of transition from co-flow to counter-flow. (a) For $A_{1}>0$ the fluid velocity $\bar{u}$ changes the sign at $\tilde{t}=T_{\mathrm{p}}$ when the cupula displacement $V$ reaches an extremum. (b) For $A_{1}<0$ the particle velocity $\partial \tilde{x}_{\mathrm{p}} / \partial \tilde{t}$ changes the sign whereas the fluid velocity and the cupula displacement decay monotonically. Solid line, $\bar{u}$; dashed line, $\partial \tilde{x}_{\mathrm{p}} / \partial \tilde{t} ;$ dot-dashed line, $V$.

Numerical simulations (figure 4) show that typical head manoeuvres always lead to co-flow situations at $\tilde{t}=0$, i.e. the endolymph and the particles move in the same direction. From figure $12 b$, however, we know that we always end up in a counter-flow configuration as $\tilde{t} \rightarrow \infty$. Therefore, either the fluid flow or the particle velocity must change direction at a certain time $\tilde{t}>0$. If $A_{1}>0$ the cupula relaxes faster than the particles fall and, therefore, the fluid velocity will change sign at $\tilde{t}=T_{\mathrm{P}}$ (figure 15). If $A_{1}<0$ the particles fall faster than the cupula relaxes. In that case, the particle velocity changes sign and there is no nystagmus. Since we were never able to observe the case $A_{1}<0$ in our numerical simulations of the full nonlinear system, we concentrate here on the case where $A_{1}>0$.

It is straightforward to show that

$$
A_{1}=\frac{\tilde{x}_{\mathrm{p}, 0}^{(1)}}{-\sigma_{\mathrm{s}} \hat{x}_{\mathrm{p}, \mathrm{s}}} \frac{\theta_{0}-\theta_{\mathrm{c}}}{\theta_{\mathrm{s}}-\theta_{\mathrm{c}}}, \quad A_{2}=-\frac{\tilde{x}_{\mathrm{p}, 0}^{(1)}}{-\sigma_{\mathrm{c}} \hat{x}_{\mathrm{p}, \mathrm{c}}} \frac{\theta_{0}-\theta_{\mathrm{s}}}{\theta_{\mathrm{s}}-\theta_{\mathrm{c}}},
$$

where $\theta_{0}=\bar{u}_{0} / \tilde{x}_{\mathrm{p}, 0}^{(1)}$ is the ratio between the initial fluid and particle velocities. The values $\theta_{\mathrm{c}}$ and $\theta_{\mathrm{s}}$ were discussed in $§ 5.1$. From figure 15 and the expression for $A_{1}$ it becomes clear that we get a positional nystagmus if and only if $\theta_{0}<\theta_{\mathrm{c}}$. The time of peak nystagmus $T_{\mathrm{P}}$ corresponds to the time where $\bar{u}$ changes sign (cf. figure 15a). From (5.10), we can compute it as

$$
T_{\mathrm{P}}=\frac{1}{\sigma_{\mathrm{c}}-\sigma_{\mathrm{s}}} \ln \left[\frac{\theta_{\mathrm{c}}\left(\theta_{0}-\theta_{\mathrm{s}}\right)}{\theta_{\mathrm{s}}\left(\theta_{0}-\theta_{\mathrm{c}}\right)}\right] .
$$

Note that $T_{\mathrm{P}}$ goes to infinity as $\theta_{0} \rightarrow \theta_{\mathrm{c}}$. Similarly, we can find an expression for $T_{\mathrm{L}}$. And the magnitude of the 


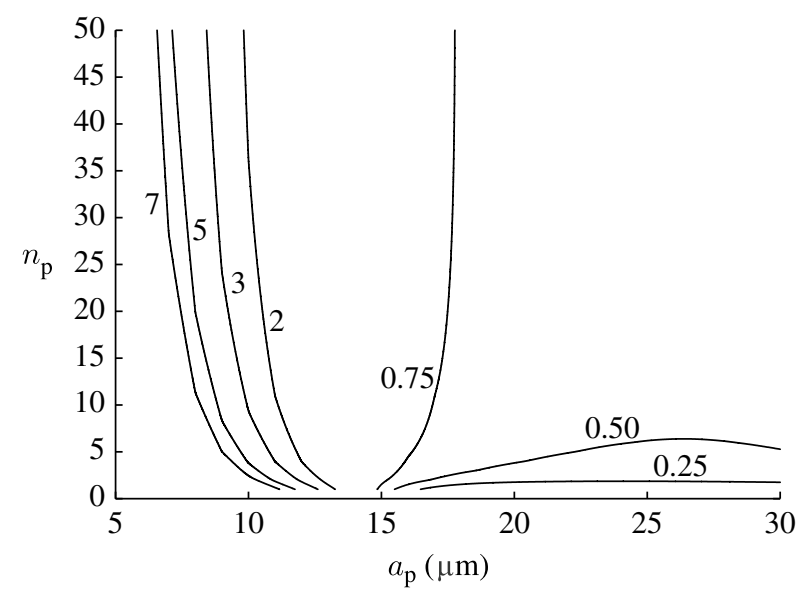

Figure 16. Value of $1-\theta_{0} / \theta_{\mathrm{u}}$ as a function of the particle size $a_{\mathrm{p}}$ and number $n_{\mathrm{p}}$ (values indicated at the contour lines).

maximum cupula displacement is

$$
V_{\max }=A_{\mathrm{c}} \int_{T_{\mathrm{P}}}^{\infty} \bar{u}(\tilde{t}) \mathrm{d} \tilde{t},
$$

which gives

$$
V_{\max }=A_{\mathrm{c}} \tilde{x}_{\mathrm{p}, 0}^{(1)}\left(\frac{1}{\sigma_{\mathrm{s}}}-\frac{1}{\sigma_{\mathrm{c}}}\right) \theta_{\mathrm{s}} \frac{\theta_{0}-\theta_{\mathrm{c}}}{\theta_{\mathrm{s}}-\theta_{\mathrm{c}}}\left[\frac{\theta_{\mathrm{c}}\left(\theta_{0}-\theta_{\mathrm{s}}\right)}{\theta_{\mathrm{s}}\left(\theta_{0}-\theta_{\mathrm{c}}\right)}\right]^{-\sigma_{\mathrm{s}} /\left(\sigma_{\mathrm{c}}-\sigma_{\mathrm{s}}\right)} .
$$

We can simplify these expressions using our results from $\S 4$ and figures $12 b$ and 13

$$
\text { For } S t>1: \quad S t \approx \frac{\sigma_{\mathrm{c}}}{\sigma_{\mathrm{s}}},\left|\theta_{\mathrm{c}}\right| \gg\left|\theta_{0}\right|, \quad\left|\theta_{\mathrm{c}}\right| \gg\left|\theta_{\mathrm{s}}\right|, \quad \theta_{\mathrm{s}} \approx \theta_{\mathrm{u}}
$$

For $S t<1: \quad S t \approx \frac{\sigma_{\mathrm{s}}}{\sigma_{\mathrm{c}}}, \quad\left|\theta_{\mathrm{s}}\right| \gg\left|\theta_{0}\right|, \quad\left|\theta_{\mathrm{s}}\right| \gg\left|\theta_{\mathrm{c}}\right|, \quad \theta_{\mathrm{c}} \approx \theta_{\mathrm{u}}$

This yields the following expressions for the onset latency $T_{\mathrm{L}}$, the time to peak $T_{\mathrm{P}}$ and the magnitude of the maximum cupula displacement $V_{\max }$

$$
\begin{gathered}
T_{\mathrm{P}}=\frac{16}{\epsilon} \frac{S t}{S t-1} \ln \left(1-\frac{\theta_{0}}{\theta_{\mathrm{u}}}\right), \\
T_{\mathrm{P}}-T_{\mathrm{L}}=\frac{16}{\epsilon} \frac{S t}{S t-1} \ln S t, \\
V_{\max }=\frac{16}{\epsilon} \bar{u}_{0}(S t-1)\left(1-\frac{\theta_{0}}{\theta_{\mathrm{u}}}\right)^{-1 /(S t-1)} .
\end{gathered}
$$

Typical values for $1-\theta_{0} / \theta_{\mathrm{u}}$ can be found in figure 16 .

Although only approximately valid for the linearized equations, these explicit expressions give us a good idea of the relationship between the physical and geometrical properties and the characteristic features of the positional nystagmus. For instance, we see that all characteristic values scale linearly with the cupula time constant $16 / \epsilon$. We also find that the time from the onset of the nystagmus to its peak depends strongly on the Stokes number $S t$, but that it is independent (in the spirit of a first approximation) of the head manoeuvre and the number of particles.

\section{REFERENCES}

Aw, S., Todd, M., Aw, G., Mcgarvie, L. \& Halmagyi, G. 2005 Benign positional nystagmus - a study of its three-dimensional spatio-temporal characteristics. Neurology 64, 1897-1905. (doi:10.1212/01.WNL.0000163545. 57134.3D)

Baloh, R. W., Sloane, P. D. \& Honrubia, V. 1989 Quantitative vestibular function testing in elderly patients with dizziness. Ear Nose Throat J. 68, 935-939.

Breuer, J. 1874 Über die Funktion der Bogengänge des Ohrlabyrinths. Wien. Med. Jahrb. 4, 72.

Bungay, P. M. \& Brenner, H. 1973 Pressure drop due to the motion of a sphere near the wall bounding a Poiseuille flow. J. Fluid Mech. 60, 81-96. (doi:10.1017/S0022112 073000054)

Campos, A. 1990 Otoconial degeneration in the aged utricle and saccule. Adv. Otorhinol. 45, 143-153.

Clift, R., Grace, J. \& Weber, M. 1978 Bubbles, drops and particles. London, UK: Academic Press.

Cohen, B., Matsuo, V. \& Raphan, T. 1977 Quantitative analysis of the velocity characteristics of optokinetic nystagmus and optokinetic after-nystagmus. J. Physiol. 270, 321-344.

Colonius, T. \& Lele, S. K. 2004 Computational aeroacoustics: progress on nonlinear problems of sound generation. Prog. Aerosp. Sci. 40, 345-416. (doi:10.1016/j.paerosci. 2004.09.001)

Crum Brown, A. 1874 On the sense of rotation and the anatomy and physiology of the semicircular canals of the inner ear. J. Anat. Physiol. 8, 327.

Dai, M., Klein, A., Cohen, B. \& Raphan, T. 1999 Model-based study of the human cupular time constant. J. Vest. Res. 9, 293-301.

Damiano, E. \& Rabbitt, R. 1996 A singular perturbation model of fluid dynamics in the vestibular semicircular canal and ampulla. J. Fluid Mech. 307, 333-372. (doi:10. 1017/S0022112096000146)

Dix, M. \& Hallpike, C. 1952 The pathology, symptomatology, and diagnosis of certain common disorders of the vestibular system. Proc. R. Soc. Med. 45, 341-354.

Ferziger, J. H. \& Perić, M. 2002 Computational methods for fluid dynamics, 3rd edn. Berlin, Germany: Springer.

Happel, J. \& Brenner, H. 1973 Low Reynolds number hydrodynamics: with special applications to particulate media, 2nd edn. Leyden, Germany: Noordhoff.

Hepp, K. 1990 On Listing's law. Commun. Math. Phys. 132, 285-292. (doi:10.1007/BF02278012)

House, M. G. \& Honrubia, V. 2003 Theoretical models for the mechanisms of benign paroxysmal positional vertigo. Audiol. Neurootol. 8, 91-99. (doi:10.1159/000068998)

Ifediba, M. A., Rajguru, S. M., Hullar, T. E. \& Rabbitt, R. D. 2007 The role of 3-canal biomechanics in angular motion transduction by the human vestibular labyrinth. Ann. Biomed. Eng. 35, 1247-1263. (doi:10.1007/s10439-0079277-y)

Mach, E. 1875 Grundlinien der Lehre von den Bewegungsempfindungen. Leipzig, Germany: Wilhelm Engelmann.

Obrist, D. 2008 Fluidmechanics of semicircular canalsrevisited. Z. Angew. Math. Phys 59, 475-497. (doi:10.1007/ s00033-007-6037-7)

Rabbitt, R. \& Damiano, E. 1992 A hydroelastic model of macromechanics in the endolymphatic vestibular canal. J. Fluid Mech. 238, 337-369. (doi:10.1017/S0022112092 001745)

Rajguru, S. M. \& Rabbitt, R. D. 2007 Afferent responses during experimentally induced semicircular canalithiasis. J. Neurophysiol. 97, 2355-2363. (doi:10.1152/jn.01152. 2006) 
Rajguru, S. M., Ifediba, M. A. \& Rabbitt, R. D. 2004 Threedimensional biomechanical model of benign paroxysmal positional vertigo. Ann. Biomed. Eng. 32, 831-846. (doi:10.1023/B:ABME.0000030259.41143.30)

Rajguru, S. M., Ifediba, M. A. \& Rabbitt, R. D. 2005 Biomechanics of horizontal canal benign paroxysmal positional vertigo. J. Vest. Res. 15, 203-214.

Raphan, T., Matsuo, V. \& Cohen, B. 1979 Velocity storage in the vestibulo-ocular reflex arc (VOR). Exp. Brain Res. 35, 229-248. (doi:10.1007/BF00236613)

Robinson, D. 1977 Linear addition of optokinetic and vestibular signals in the vestibular nucleus. Exp. Brain Res. 30, 447-450. (doi:10.1007/BF00237269)

Squires, T. M., Weidmann, M. S., Hain, T. C. \& Stone, H. A. 2004 A mathematical model for top-shelf vertigo: the role of sedimenting otoconia in BPPV. J. Biomech. 37, 1137-1146. (doi:10.1016/j.jbiomech.2003.12.014)
Tweed, D., Cadera, W. \& Vilis, T. 1990 Computing threedimensional eye position quaternions and eye velocity from search coil signals. Vision Res. 30, 97-110. (doi:10. 1016/0042-6989(90)90130-D)

Van Buskirk, W. C. 1977 The effects of the utricle on fluidflow in semicircular canals. Ann. Biomed. Eng. 5, 1-11. (doi:10.1007/BF02409335)

Van Buskirk, W. \& Grant, J. 1973 Biomechanics of the semicircular canals. In Biomechanics symposium, pp. 53-54. New York, NY: ASME.

Van Buskirk, W., Watts, R. \& Liu, Y. 1976 The fluid mechanics of the semicircular canals. J. Fluid Mech. 78, 87-98. (doi:10.1017/S0022112076002346)

Yamauchi, A., Rabbitt, R., Boyle, R. \& Highstein, S. 2001 Relationship between inner-ear fluid pressure and semicircular canal afferent nerve discharge. J. Assoc. Res. Otolaryngol. 3, 24-44. 